\title{
Stabilizability in optimal control
}

\author{
Anna Chiara Lai and Monica Motta(i)
}

\begin{abstract}
We extend the classical concepts of sampling and Euler solutions for control systems associated to discontinuous feedbacks by considering also the corresponding costs. In particular, we introduce the notions of Sample and Euler stabilizability to a closed target set $\mathbf{C}$ with $\left(p_{0}, W\right)$ regulated cost, for some continuous, state-dependent function $W$ and some constant $p_{0}>0$ : it roughly means that we require the existence of a stabilizing feedback $K$ such that all the corresponding sampling and Euler solutions starting from a point $z$ have suitably defined finite costs, bounded above by $W(z) / p_{0}$. Then, we show how the existence of a special, semiconcave Control Lyapunov Function $W$, called $p_{0}$-Minimum Restraint Function, allows us to construct explicitly such a feedback $K$. When dynamics and Lagrangian are Lipschitz continuous in the state variable, we prove that $K$ as above can be still obtained if there exists a $p_{0}$-Minimum Restraint Function which is merely Lipschitz continuous. An example on the stabilizability with $\left(p_{0}, W\right)$-regulated cost of the nonholonomic integrator control system associated to any cost with bounded Lagrangian illustrates the results.
\end{abstract}

Mathematics Subject Classification. 93D05, 93D20, 49J15.

Keywords. Asymptotic stabilizability, Discontinuous feedback law, Optimal control, Exit-time problems.

\section{Introduction}

In this paper we investigate in an optimal control perspective the stabilizability to a set $\mathbf{C} \subset \mathbb{R}^{n}$ of the nonlinear control system

$$
\dot{x}=f(x, u), \quad u \in U,
$$

This research is partially supported by the Padua University grant SID 2018 "Controllability, stabilizability and infimum gaps for control systems", prot. BIRD 187147, and by the Gruppo Nazionale per l' Analisi Matematica, la Probabilità e le loro Applicazioni (GNAMPA) of the Istituto Nazionale di Alta Matematica (INdAM), Italy. 
to which we associate a cost of the form

$$
\int_{0}^{T_{x}} l(x(\tau), u(\tau)) d \tau
$$

where $l \geq 0$, and $T_{x} \leq+\infty$ - the exit-time of $x$-verifies

$$
x(t) \in \mathbb{R}^{n} \backslash \mathbf{C} \text { for all } t \in\left[0, T_{x}\right), \quad \lim _{t \rightarrow T_{x}^{-}} \mathbf{d}(x(t))=0
$$

(for any $y \in \mathbb{R}^{n}, \mathbf{d}(y)$ denotes the distance of $y$ from $\mathbf{C}$ ). In particular, for any $r>0$, we set $B_{r}(\mathbf{C}):=\left\{y \in \mathbb{R}^{n}: \mathbf{d}(y) \leq r\right\}$ and assume that:

(H0) the target set $\mathbf{C} \subset \mathbb{R}^{n}$ is closed, with compact boundary; the control set $U \subseteq \mathbb{R}^{m}$ is closed, not necessarily bounded; the functions $f:\left(\mathbb{R}^{n} \backslash \mathbf{C}\right) \times U \rightarrow$ $\mathbb{R}^{n}, l:\left(\mathbb{R}^{n} \backslash \mathbf{C}\right) \times U \rightarrow[0,+\infty)$ are uniformly continuous on $\mathcal{K} \times U$ for any compact set $\mathcal{K} \subset \mathbb{R}^{n} \backslash \mathbf{C}$; for any $R>0$ there is some $M(R)>0$ such that

$$
|f(x, u)| \leq M(R), \quad l(x, u) \leq M(R) \quad \forall(x, u) \in\left(B_{R}(\mathbf{C}) \backslash \mathbf{C}\right) \times U .
$$

Hence, for any admissible control, given by a function $u \in L_{l o c}^{\infty}\left(\left[0, T_{x}\right), U\right){ }^{1}$ every Cauchy problem associated to (1.1) has in general multiple solutions and the cost may be finite even if $T_{x}=+\infty$.

In order to obtain sufficient conditions for the stabilizability of the system with regulated cost, we consider the Hamiltonian

$$
H\left(x, p_{0}, p\right):=\inf _{u \in U}\left\{\langle p, f(x, u)\rangle+p_{0} l(x, u)\right\},
$$

and the following notion, firstly introduced in [23] (in a slightly weaker form).

Definition 1.1. ( $p_{0}$-Minimum Restraint Function) Let $W: \overline{\mathbb{R}^{n} \backslash \mathbf{C}} \rightarrow[0,+\infty)$ be a continuous function, which is locally semiconcave, positive definite, and proper on $\mathbb{R}^{n} \backslash \mathbf{C}$. We say that $W$ is a $p_{0}$-Minimum Restraint Function-in short, $p_{0}-M R F$ - for some $p_{0} \geq 0$, if it verifies the decrease condition:

$$
H\left(x, p_{0}, D^{*} W(x)\right) \leq-\gamma(W(x)) \quad \forall x \in \mathbb{R}^{n} \backslash \mathbf{C},
$$

2 for some continuous, strictly increasing function $\gamma:(0,+\infty) \rightarrow(0,+\infty)$.

A $p_{0}$-Minimum Restraint Function is at once a Control Lyapunov Function for (1.1) and, by [23, Prop. 5.1], also a strict viscosity supersolution to the Hamilton-Jacobi-Bellman equation

$$
\sup _{u \in U}\left\{-\langle D W(x), f(x, u)\rangle-p_{0} l(x, u)\right\}-\gamma(W(x))=0, \quad x \in \mathbb{R}^{n} \backslash \mathbf{C} .
$$

Hence, when a $p_{0}$-Minimum Restraint Function exists, on the one hand, the (open-loop) global asymptotic controllability of the control system (1.1) to C-namely, that for any initial condition $z \in \mathbb{R}^{n} \backslash \mathbf{C}$ there is an admissible trajectory-control pair $(x, u)$ to $(1.1)$ with $x(0)=z$, such that $\lim _{t \rightarrow T_{x}^{-}} \mathbf{d}(x(t))=$

\footnotetext{
${ }^{1}$ That is, $u:\left[0, T_{x}\right) \rightarrow U$ is a measurable function which is essentially bounded on any subinterval $[0, T] \subset\left[0, T_{x}\right)$.

${ }^{2}$ This means that $H\left(x, p_{0}, p\right) \leq-\gamma(W(x))$ for every $p \in D^{*} W(x)$, where $D^{*} W(x)$ denotes the set of reachable gradients of $W$ at $x$ (see Sect. 1.1).
} 
0 in a certain uniform and stable manner that we will not dwell upon here - is expected (see e.g. $[3,30,32]$ ). On the other hand, if $p_{0}>0$, the existence of an admissible trajectory-control pair $(x, u)$ with $x(0)=z$ satisfying the cost estimate

$$
\int_{0}^{T_{x}} l(x(t), u(t)) d t \leq \frac{W(z)}{p_{0}}
$$

follows by known optimality principles (see e.g. [22,25,33]). The main contribution of $[19,23]$ was to prove that the existence of a $p_{0}$-Minimum Restraint Function $W$ for some $p_{0}>0$ allows to produce a pair $(x, u)$ that meets both of these properties.

A related and important goal is, given a $p_{0}$-Minimum Restraint Function $W$ for some $p_{0}>0$, to provide a state feedback $K: \mathbb{R}^{n} \backslash \mathbf{C} \rightarrow U$ such that the system $\dot{x}(t)=f(x(t), K(x(t))$ is globally asymptotically stable to $\mathbf{C}$ and has $\left(p_{0}, W\right)$-regulated cost, that is, such that for any stable trajectory $x$ with $x(0)=z$, the cost $\int_{0}^{T_{x}} l(x(t), K(x(t))) d t$ is not greater than $W(z) / p_{0}$. In this paper we address the question, left by $[19,23]$ as an open problem, of how to define such a feedback law through the use of $W$. In the ideal case in which $W$ is differentiable and there exists a continuous feedback $K(x)$ such that $\langle D W(x), f(x, K(x))\rangle+p_{0} l(x, K(x)) \leq-\gamma(W(x))$ for all $x \in \mathbb{R}^{n} \backslash$ $\mathbf{C}$, one easily derives global asymptotic stabilizability with $\left(p_{0}, W\right)$-regulated cost. However, it is a classical matter in nonlinear control systems that a differentiable Control Lyapunov Function $W$ may not exist and, even if a smooth $W$ exists, a continuous feedback $K$ does not generally exist (see e.g. [1, $7,15,30-32]$ ). From here, the need of considering the nonsmooth version (1.6) of the decrease condition and of defining a discontinuous feedback $K: \mathbb{R}^{n} \backslash \mathbf{C} \rightarrow U$, which, because of the unboundedness of $U$, we can only assume locally bounded (see Prop. 3.2 below). In particular, we might have $\lim \sup _{x \rightarrow \bar{x} \in \partial \mathbf{C}}|K(x)|=$ $+\infty$.

Identified a feedback $K$ as above, the main issue is how to interpret the discontinuous differential equation and the associated exit-time cost, so that the control system $(1.1)$ can be stabilized to the target $\mathbf{C}$ with $\left(p_{0}, W\right)$ regulated cost by $K$. For the trajectories of $\dot{x}(t)=f(x(t), K(x(t))$, we simply adapt to our setting the nowadays classical notions of sampling and Euler solutions in $[13,14]$, inspired by differential games theory [17]. However, our primary objective is to introduce a suitable concept of cost associated to a stable sampling or Euler solution starting from $z$, so that such cost is bounded above by $W(z) / p_{0}$. Postponing the precise definitions to Sect. 2, given some constants $r, R$ such that $0<r<R$, we call a sampling pair $(x, u)(r, R)$-stable when, starting from some $z$ with $r<\mathbf{d}(z) \leq R, x$ reaches in a uniform manner the $r$-neighborhood $B_{r}(\mathbf{C})$ of the target and, after a time $\bar{T}_{x}^{r}$, remains definitely in $B_{r}(\mathbf{C})$. In this case, for all $t \geq 0$ we define the corresponding sampling cost as

$$
x^{0}(t):=\int_{0}^{\min \left\{t, \bar{T}_{x}^{r}\right\}} l(x(s), u(s)) d s
$$


and show that $x^{0}(t) \leq W(z) / p_{0}$. The difficulty in proving the latter inequality lies in the fact that $\overline{\bar{T}}_{x}^{r}$ may not be the first instant in which $x$ enters $B_{r}(\mathbf{C})$. Consequently, we must estimate the cost in a time interval where we basically have no information on $x$, except that it is in $B_{r}(\mathbf{C})$ (see Sect. 3.1 below). Nevertheless, this is the correct notion of sampling cost. Indeed, let us now define an Euler cost-solution pair $\left(X^{0}, X\right)$ as the locally uniform limit on $[0,+\infty)$ of a sequence of sampling cost-trajectory pairs as above, when the sampling times tend to zero. We obtain that $\mathcal{X}$ approaches uniformly asymptotically $\mathbf{C}$, while $\lim _{t \rightarrow T_{x}^{-}} X^{0}(t) \leq W(z) / p_{0}$, where $T_{X} \leq+\infty$ is the exit-time of $X$, as in (1.3) (see Sect. 3.2).

Furthermore, inspired by [28], we prove that, when $f$ and $l$ are locally Lipschitz continuous in $x$ uniformly w.r.t. $u \in U$ up to the boundary of $\mathbf{C}$, the existence of a $p_{0}$-Minimum Restraint Function $W$ with $p_{0}>0$, possibly not locally semiconcave but merely locally Lipschitz continuous on $\overline{\mathbb{R}^{n} \backslash \mathbf{C}}$, still guarantees stabilizability of $(1.1)$ to $\mathbf{C}$ with $\left(p_{0}, W\right)$-regulated cost, in the sample and Euler sense (see Theorem 4.3 and Corollary 4.4). This result can be useful for the effective implementation of our feedback construction, as shown by the example in Sect. 5 .

We are motivated to consider $U$ unbounded and the minimization in the decrease condition on the whole set $U$ (and not on bounded subsets, as usual) mainly because these assumptions are met, for instance, in the stabilization of mechanical systems with vibrating controls. These are nonlinear control systems, affine or quadratic in the derivative of the control, which is considered as an impulsive control (see [6]). In particular, the reparameterized systems usually introduced in the study of control-polynomial systems satisfy (H0). As a consequence, as shown in [18], the results of the present paper are the starting point for the stabilizability (with and without regulated cost) of impulsive control systems. In fact, our assumptions include also the case where $U$ is bounded and $f$ and $l$ are continuous on $\mathbb{R}^{n} \times U$. For $U$ unbounded, they are satisfied, for instance, by the class of control problems in which the input appears inside a saturation nonlinearity, such as $f(x, u)=f_{0}(x)+\sum_{i=1}^{m} f_{i}(x) \sigma_{i}(u), l(x, u)=l_{0}(x)+l_{1}(x)\left|\sigma_{0}(u)\right|$, where $l_{0}$, $l_{1}, f_{0}, f_{1}, \ldots, f_{m} \in C\left(\mathbb{R}^{n}\right)$ and $\sigma_{0}, \ldots, \sigma_{m}$ are bounded, uniformly continuous maps on $U$. The stabilizability of such control systems plays a relevant role both in the literature and in the applications (see e.g. [4,10,11,34]).

Finally, the value function

$$
V(z):=\inf _{(x, u), x(0)=z} \int_{0}^{T_{x}} l(x(\tau), u(\tau)) d \tau
$$

is clearly bounded above by any $p_{0}$-Minimum Restraint Function divided by $p_{0}$. Hence our approach could be useful to design approximated optimal closedloop strategies, when there exists a sequence of $p_{0}$-Minimum Restraint Functions approaching $V$, as in [25], or at least to obtain "safe" performances, keeping the cost under the value $W$. Moreover, when $V \leq W / p_{0}$, then $V$ 
is continuous on the target's boundary and this is crucial to establish comparison, uniqueness, and robustness properties for the associated HamiltonJacobi-Bellman equation $[22,24,25]$ and to study associated asymptotic and ergodic problems [26]. From this PDE point of view, problem (1.1)-(1.2) has been widely investigated; a likely incomplete bibliography, also containing applications (for instance, the Füller and shape-from-shading problems), includes $[8,16,20,33]$ and the references therein.

The paper is organized as follows. In the remaining part of the Introduction we provide some preliminary definitions and notation. In Sect. 2 we define precisely the sample and Euler stabilizability of (1.1) to $\mathbf{C}$ with $\left(p_{0}, W\right)$-regulated cost, which is shown to be guaranteed by the existence of a $p_{0}$-Minimum Restraint Function in Theorem 3.1, our main result. In Sect. 4 we consider the case of Lipschitz continuous data, postponing the proofs in the "Appendix". An example on the stabilizability with regulated cost of the non-holonomic integrator control system concludes the paper (see Sect. 5).

\subsection{Notation and preliminaries}

For every $r \geq 0$ and $\Omega \subset \mathbb{R}^{N}$, we set $B_{r}(\Omega):=\left\{x \in \mathbb{R}^{N} \mid d(x, \Omega) \leq r\right\}$, where $d$ is the usual Euclidean distance. When $\Omega=\{z\}$ for some $z \in \mathbb{R}^{N}$, we also use of the notation $B(z, r):=B_{r}(\{z\})$. For any map $F: \Omega \rightarrow \mathbb{R}^{M}$ we call modulus (of continuity) of $F$ any increasing, continuous function $\omega:[0,+\infty) \rightarrow[0,+\infty)$ such that $\omega(0)=0, \omega(r)>0$ for every $r>0$ and $\left|F\left(x_{1}\right)-F\left(x_{2}\right)\right| \leq \omega\left(\left|x_{1}-x_{2}\right|\right)$ for all $x_{1}, x_{2} \in \Omega$. We use $\bar{\Omega}, \stackrel{\circ}{\Omega}$ to denote the closure and the interior of the set $\Omega$, respectively.

Let us summarize some basic notions in nonsmooth analysis (see e.g. $[9,12,35]$ for a thorough treatment).

Definition 1.2. (Positive definite and proper functions). Let $\Omega \subset \mathbb{R}^{N}$ be an open set with compact boundary. A continuous function $F: \bar{\Omega} \rightarrow \mathbb{R}$ is said positive definite on $\Omega$ if $F(x)>0 \forall x \in \Omega$ and $F(x)=0 \forall x \in \partial \Omega$. The function $F$ is called proper on $\Omega$ if the pre-image $F^{-1}(K)$ of any compact set $K \subset[0,+\infty)$ is compact.

Definition 1.3. (Semiconcavity). Let $\Omega \subseteq \mathbb{R}^{N}$. A continuous function $F: \Omega \rightarrow$ $\mathbb{R}$ is said to be semiconcave on $\Omega$ if there exists $\rho>0$ such that

$$
F(x)+F(\hat{x})-2 F\left(\frac{x+\hat{x}}{2}\right) \leq \rho|x-\hat{x}|^{2},
$$

for all $x, \hat{x} \in \Omega$ such that $[x, \hat{x}] \subset \Omega$. The constant $\rho$ above is called a semiconcavity constant for $F$ in $\Omega$. $F$ is said to be locally semiconcave on $\Omega$ if it semiconcave on every compact subset of $\Omega$.

We remind that locally semiconcave functions are locally Lipschitz continuous. Actually, they are twice differentiable almost everywhere.

Definition 1.4. (Limiting gradient). Let $\Omega \subseteq \mathbb{R}^{N}$ be an open set, and let $F: \Omega \rightarrow \mathbb{R}$ be a locally Lipschitz continuous function. For every $x \in \Omega$ we call 
set of limiting gradients of $F$ at $x$, the set:

$D^{*} F(x):=\left\{w \in \mathbb{R}^{N}: \quad w=\lim _{k} \nabla F\left(x_{k}\right), \quad x_{k} \in D I F F(F) \backslash\{x\}, \quad \lim _{k} x_{k}=x\right\}$,

where $\nabla$ denotes the classical gradient operator and $\operatorname{DIFF}(F)$ is the set of differentiability points of $F$.

The set-valued map $x \rightsquigarrow D^{*} F(x)$ is upper semicontinuous on $\Omega$, with nonempty, compact, and not necessarily convex values. When $F$ is a locally semiconcave function, $D^{*} F$ coincides with the limiting subdifferential $\partial_{L} F$, namely,

$$
D^{*} F(x)=\partial_{L} F(x):=\left\{\lim p_{i}: p_{i} \in \partial_{P} F\left(x_{i}\right), \lim x_{i}=x\right\} \quad \forall x \in \Omega .
$$

As usual, for every $x \in \Omega$, the proximal subdifferential of $F$ at $x$ is given by

$$
\begin{aligned}
\partial_{P} F(x):=\left\{p \in \mathbb{R}^{N}: \exists \sigma, \eta>0 \text { s.t., } \forall y \in B(x, \eta),\right. \\
\left.F(y)-F(x)+\sigma|y-x|^{2} \geq\langle p, y-x\rangle\right\} .
\end{aligned}
$$

For locally Lipschitz continuous functions, the Clarke subdifferential $\partial_{C} F(x)$ of $F$ at $x$, can be defined as $\partial_{C} F(x):=\operatorname{co} \partial_{L} F(x)$. Finally, locally semiconcave functions enjoy the following properties (see [9, Propositions 3.3.1, 3.6.2]).

Lemma 1.5. Let $\Omega \subseteq \mathbb{R}^{N}$ be an open set and let $F: \Omega \rightarrow \mathbb{R}$ be a locally semiconcave function. Then for any compact set $\mathcal{K} \subset \Omega$ there exist some positive constants $L$ and $\rho$ such that, for any $x \in \mathcal{K},{ }^{3}$

$$
\begin{aligned}
& F(\hat{x})-F(x) \leq\langle p, \hat{x}-x\rangle+\rho|\hat{x}-x|^{2}, \\
& |p| \leq L \quad \forall p \in D^{*} F(x),
\end{aligned}
$$

for any point $\hat{x} \in \mathcal{K}$ such that $[x, \hat{x}] \subset \mathcal{K}$.

\section{Sample and Euler stabilizability with regulated cost}

Let us introduce the notions of sampling and Euler solutions with regulated cost. Hypothesis (H0) is assumed throughout the whole section.

Definition 2.1. (Admissible trajectory-control pairs and costs) For every point $z \in \mathbb{R}^{n} \backslash \mathbf{C}$, we will say that $(x, u)$ is an admissible trajectory-control pair from $z$ for the control system

$$
\dot{x}=f(x, u),
$$

if there exists $T_{x} \leq+\infty$ such that $u \in L_{l o c}^{\infty}\left(\left[0, T_{x}\right), U\right)$ and $x$ is a Carathéodory solution of $(2.1)$ in $\left[0, T_{x}\right)$ corresponding to $u$, verifying $x(0)=z$ and

$$
x\left(\left[0, T_{x}\right)\right) \subset \mathbb{R}^{n} \backslash \mathbf{C} \text { and, if } T_{x}<+\infty, \lim _{t \rightarrow T_{x}^{-}} \mathbf{d}(x(t))=0 .
$$

\footnotetext{
${ }^{3}$ The inequality (1.8) is usually formulated with the proximal superdifferential $\partial^{P} F$. However, this does not make a difference here since $\partial^{P} F=\partial_{C} F=c o D^{*} F$ as soon as $F$ is locally semiconcave. Hence (1.8) is true in particular for any $p \in D^{*} F(x)$.
} 
We shall use $\mathcal{A}_{f}(z)$ to denote the family of admissible trajectory-control pairs $(x, u)$ from $z$ for the control system (2.1). Moreover, we will call cost associated to $(x, u) \in \mathcal{A}_{f}(z)$ the function

$$
x^{0}(t):=\int_{0}^{t} l(x(\tau), u(\tau)) d \tau \quad \forall t \in\left[0, T_{x}\right) .
$$

If $T_{x}<+\infty$, we extend continuously $\left(x^{0}, x\right)$ to $[0,+\infty)$, by setting

$$
\left(x^{0}, x\right)(t)=\lim _{\tau \rightarrow T_{x}^{-}}\left(x^{0}, x\right)(\tau) \quad \forall t \geq T_{x} .
$$

From now on, we will always consider admissible trajectories and associated costs defined on $[0,+\infty)$.

Observe that for any admissible trajectory-control pair defined on $\left[0, T_{x}\right)$, when $T_{x}<+\infty$ the above limit exists by (H0). In particular, this follows by the compactness of $\partial \mathbf{C}$ and the boundedness of $f$ and $l$ in any bounded neighborhood of the target.

A partition of $[0,+\infty)$ is a sequence $\pi=\left(t^{j}\right)$ such that $t^{0}=0, \quad t^{j-1}<t^{j}$ $\forall j \geq 1$, and $\lim _{j \rightarrow+\infty} t^{j}=+\infty$. The value $\operatorname{diam}(\pi):=\sup _{j \geq 1}\left(t^{j}-t^{j-1}\right)$ is called the diameter or the sampling time of the sequence $\pi$. A feedback for (2.1) is defined to be any locally bounded function $K: \mathbb{R}^{n} \backslash \mathbf{C} \rightarrow U$.

Definition 2.2. (Sampling trajectory and sampling cost) Given a feedback $K: \mathbb{R}^{n} \backslash \mathbf{C} \rightarrow U$, a partition $\pi=\left(t^{j}\right)$ of $[0,+\infty)$, and a point $z \in \mathbb{R}^{n} \backslash \mathbf{C}$, a $\pi$-sampling trajectory for (2.1) from $z$ associated to $K$ is a continuous function $x$ defined by recursively solving

$$
\dot{x}(t)=f\left(x(t), K\left(x\left(t^{j-1}\right)\right) \quad t \in\left[t^{j-1}, t^{j}\right],\left(x(t) \in \mathbb{R}^{n} \backslash \mathbf{C}\right)\right.
$$

from the initial time $t^{j-1}$ up to time

$$
\tau^{j}:=t^{j-1} \vee \sup \left\{\tau \in\left[t^{j-1}, t^{j}\right]: x \text { is defined on }\left[t^{j-1}, \tau\right)\right\},
$$

where $x\left(t^{0}\right)=x(0)=z$. In this case, the trajectory $x$ is defined on the rightopen interval from time zero up to time $t^{-}:=\inf \left\{\tau^{j}: \tau^{j}<t^{j}\right\}$. Accordingly, for every $j \geq 1$, we set

$$
u(t):=K\left(x\left(t^{j-1}\right)\right) \quad \forall t \in\left[t^{j-1}, t^{j}\right) \cap\left[0, t^{-}\right) .
$$

The pair $(x, u)$ will be called a $\pi$-sampling trajectory-control pair of (2.1) from $z$ (corresponding to the feedback $K$ ). The sampling cost associated to $(x, u)$ is given by

$$
x^{0}(t):=\int_{0}^{t} l(x(\tau), u(\tau)) d \tau \quad t \in\left[0, t^{-}\right) .
$$

Definition 2.3. (Sample stabilizability with $\left(p_{0}, W\right)$-regulated cost) A feedback $K: \mathbb{R}^{n} \backslash \mathbf{C} \rightarrow U$ is said to sample-stabilize (2.1) to $\mathbf{C}$ if there is a function $\beta \in \mathcal{K} \mathcal{L}$ satisfying the following: for each pair $0<r<R$ there exists $\delta=\delta(r, R)>0$, such that, for every partition $\pi$ of $[0,+\infty)$ with $\operatorname{diam}(\pi) \leq \delta$ and for any initial state $z \in \mathbb{R}^{n} \backslash \mathbf{C}$ such that $\mathbf{d}(z) \leq R$, any $\pi$-sampling 
trajectory-control pair $(x, u)$ of $(2.1)$ from $z$ associated to $K$ belongs to $\mathcal{A}_{f}(z)$ and verifies:

$$
\mathbf{d}(x(t)) \leq \max \{\beta(R, t), r\} \quad \forall t \in[0,+\infty) .
$$

Such $(x, u)$ are called $(r, R)$-stable (to $\mathbf{C}$ ) sampling trajectory-control pairs. If the system (2.1) admits a sample-stabilizing feedback to $\mathbf{C}$, then it is called sample stabilizable (to $\mathbf{C}$ ).

When there exist $p_{0}>0$ and a continuous map $W: \overline{\mathbb{R}^{n} \backslash \mathbf{C}} \rightarrow[0,+\infty)$ which is positive definite and proper on $\mathbb{R}^{n} \backslash \mathbf{C}$, such that the sampling cost $x^{0}$ associated to any $(r, R)$-stable sampling pair $(x, u)$ verifies

$$
x^{0}\left(\bar{T}_{x}^{r}\right)=\int_{0}^{\bar{T}_{x}^{r}} l(x(\tau), u(\tau)) d \tau \leq \frac{W(z)}{p_{0}}
$$

where

$$
\bar{T}_{x}^{r}:=\inf \{t>0: \mathbf{d}(x(\tau)) \leq r \quad \forall \tau \geq t\},
$$

we say that $(2.1)$ is sample stabilizable (to $\mathbf{C})$ with $\left(p_{0}, W\right)$-regulated cost.

Observe that, when $\mathbf{d}(z) \leq r$, the time $\bar{T}_{x}^{r}$ may be zero. In this case $(2.5)$ imposes no conditions on the cost.

Let us now introduce Euler solutions and the associated costs and a notion of Euler stabilizability to $\mathbf{C}$ with $\left(p_{0}, W\right)$-regulated cost.

Definition 2.4. (Euler trajectory and Euler cost) Let $\left(\pi_{i}\right)$ be a sequence of partitions of $[0,+\infty)$ such that $\delta_{i}:=\operatorname{diam}\left(\pi_{i}\right) \rightarrow 0$ as $i \rightarrow \infty$. Given a feedback $K: \mathbb{R}^{n} \backslash \mathbf{C} \rightarrow U$ and $z \in \mathbb{R}^{n} \backslash \mathbf{C}$, for every $i$, let $\left(x_{i}, u_{i}\right) \in \mathcal{A}_{f}(z)$ be a $\pi_{i^{-}}$ sampling trajectory-control pair of (2.1) from $z$ associated to $K$ and let $x_{i}^{0}$ be the corresponding cost. If there exists a map $X:[0,+\infty) \rightarrow \mathbb{R}^{n}$, verifying

$$
x_{i} \rightarrow X \quad \text { locally uniformly in }[0,+\infty)
$$

we call $\mathcal{X}$ an Euler trajectory of (2.1) from $z$ (corresponding to the feedback $K)$.

If moreover there is a map $\mathcal{X}^{0}:[0,+\infty) \rightarrow[0,+\infty)$ verifying

$$
x_{i}^{0} \rightarrow X^{0} \quad \text { locally uniformly in }[0,+\infty),
$$

we call $X^{0}$ the Euler cost associated to $X$.

Remark 2.5. As Euler trajectories are not, in general, classical solutions to the control system (2.1), an Euler cost may not coincide with the integral of the Lagrangian along the corresponding Euler trajectory, for some control. Nevertheless, this is true in special situations, as, for instance, when the function $l$ is continuous, bounded and does not depend on the control, that is $l(x, u)=\tilde{l}(x)$ for all $(x, u)$. Indeed, in this case if there exists a sequence of sampling trajectories $x_{i} \rightarrow X$ locally uniformly in $[0,+\infty)$, the dominated convergence theorem implies that the associated $\operatorname{costs} x_{i}^{0}$ converge locally uniformly to the function $x^{0}$ verifying

$$
X^{0}(t)=\int_{0}^{t} \tilde{l}(X(\tau)) d \tau \text { for any } t \geq 0
$$


Indeed, fixed $t>0$, for all $s \in[0, t]$ one has

$$
\left|x_{i}^{0}(s)-X^{0}(s)\right| \leq \int_{0}^{s}\left|\tilde{l}\left(x_{i}(\tau)\right)-\tilde{l}(\mathcal{X}(\tau))\right| d \tau \leq t \omega\left(\sup _{\tau \in[0, t]}\left|x_{i}(\tau)-X(\tau)\right|\right),
$$

when $\omega$ denotes a modulus of $\tilde{l}$ on a suitable compact neighborhood of $\mathcal{X}([0, t])$. Therefore, $\sup _{s \in[0, t]}\left|x_{i}^{0}(s)-X^{0}(s)\right| \rightarrow 0$ as $i \rightarrow+\infty$, for every $t>0$.

Definition 2.6. (Euler stabilizability with $\left(p_{0}, W\right)$-regulated cost) The system (2.1) is Euler stabilizable to $\mathbf{C}$ with Euler stabilizing feedback $K$, if there exists a function $\beta \in \mathcal{K} \mathcal{L}$ such that for each $z \in \mathbb{R}^{n} \backslash \mathbf{C}$, every Euler solution $\mathcal{X}$ of (2.1) from $z$ associated to $K$ verifies

$$
\mathbf{d}(X(t)) \leq \beta(\mathbf{d}(z), t) \quad \forall t \in[0,+\infty) .
$$

When there exist some $p_{0}>0$ and a continuous map $W: \overline{\mathbb{R}^{n} \backslash \mathbf{C}} \rightarrow[0,+\infty)$ which is positive definite and proper on $\mathbb{R}^{n} \backslash \mathbf{C}$, such that every Euler cost $X^{0}$ associated to $\mathcal{X}$ verifies

$$
\lim _{t \rightarrow T_{x}^{-}} X^{0}(t) \leq \frac{W(z)}{p_{0}} \quad \forall z \in \mathbb{R}^{n} \backslash \mathbf{C},
$$

where

$$
T_{X}:=\inf \left\{\tau \in(0,+\infty]: X([0, \tau)) \subset \mathbb{R}^{n} \backslash \mathbf{C}, \quad \lim _{t \rightarrow \tau^{-}} \mathbf{d}(X(t))=0\right\},
$$

then $(2.1)$ is said to have a $\left(p_{0}, W\right)$-regulated cost (w.r.t. the feedback $\left.K\right)$.

\section{Main result}

Theorem 3.1. Assume hypothesis $(\mathrm{H} 0)$ and let $W$ be a $p_{0}-M R F$ with $p_{0}>0$. Then there exists a locally bounded feedback $K: \mathbb{R}^{n} \backslash \mathbf{C} \rightarrow U$ that sample and Euler stabilizes system (2.1) to $\mathbf{C}$ with $\left(p_{0}, W\right)$-regulated cost.

We split the proof of Theorem 3.1 in two subsections, concerning with the sample stabilizability and the Euler stabilizability, respectively.

Preliminarily, let us observe that for any $(x, p) \in\left(\mathbb{R}^{n} \backslash \mathbf{C}\right) \times \mathbb{R}^{n}$ the infimum in the definition of the Hamiltonian $H$ can be taken over a compact subset of $U$, in view of the following result.

Proposition 3.2. Assume $(\mathrm{H} 0)$ and let $W$ be a $p_{0}-M R F$ with $p_{0} \geq 0$. Then there exists a continuous function $N:(0,+\infty) \rightarrow(0,+\infty)$ such that, setting for any $(x, p) \in\left(\mathbb{R}^{n} \backslash \mathbf{C}\right) \times \mathbb{R}^{n}$,

$$
H_{N(r)}\left(x, p_{0}, p\right):=\min _{u \in U \cap B(0, N(r))}\left\{\langle p, f(x, u)\rangle+p_{0} l(x, u)\right\} \quad \forall r>0
$$

one has

$$
H_{N(W(x))}\left(x, p_{0}, D^{*} W(x)\right)<-\gamma(W(x)) \quad \forall x \in \mathbb{R}^{n} \backslash \mathbf{C} .
$$


Proof. Fix $\sigma>0$. By [19, Prop. 3.3] we derive that there exists a decreasing, continuous function $N:(0, \sigma] \rightarrow(0,+\infty)$ such that, setting

$$
H_{N(r)}\left(x, p_{0}, p\right):=\min _{u \in U \cap B(0, N(r))}\left\{\langle p, f(x, u)\rangle+p_{0} l(x, u)\right\}
$$

for all $r \in(0, \sigma]$, it follows that

$$
H_{N(W(x))}\left(x, p_{0}, D^{*} W(x)\right)<-\gamma(W(x))
$$

for every $x \in W^{-1}((0, \sigma])$. It only remains to show that there exists a continuous map $N:[\sigma,+\infty) \rightarrow(0,+\infty)$ such that extending (3.2) to $r \in[\sigma,+\infty)$ one gets (3.3) for every $x \in W^{-1}([\sigma,+\infty))$. Arguing as in the proof of [19, Prop. 3.3], one can obtain that for any $r>\sigma$ there is some $N(r) \geq N(\sigma)$ such that

$$
H_{N(r)}\left(x, p_{0}, p\right)<-\gamma(W(x)) \quad \forall x \in W^{-1}([\sigma, r]) \text { and } p \in D^{*} W(x) .
$$

Moreover, for any $r_{2}>r_{1} \geq \sigma$, one clearly has $N\left(r_{2}\right) \geq N\left(r_{1}\right)$ and, enlarging $N$ if necessary, one can assume that $r \mapsto N(r)$ is increasing and continuous on $[\sigma,+\infty)$. Therefore for any $x \in W^{-1}([\sigma,+\infty))$ the thesis (3.3) follows from (3.2) as soon as $r=W(x)$.

As an immediate consequence of Proposition 3.2, the existence of a $p_{0^{-}}$ MRF $W$ guarantees the existence of a feedback $K$ with the following properties.

Proposition 3.3. Let $W$ be a $p_{0}-M R F$ with $p_{0} \geq 0$ and fix a selection $p(x) \in$ $D^{*} W(x)$ for any $x \in \mathbb{R}^{n} \backslash \mathbf{C}$. Given a function $N$ as in Proposition 3.2 , then any map $K: \mathbb{R}^{n} \backslash \mathbf{C} \rightarrow U$ such that

$$
K(x) \in \underset{u \in U \cap B(0, N(W(x))}{\arg \min }\left\{\langle p(x), f(x, u)\rangle+p_{0} l(x, u)\right\},
$$

verifies

$$
\langle p(x), f(x, K(x))\rangle+p_{0} l(x, K(x))<-\gamma(W(x)) \quad \forall x \in \mathbb{R}^{n} \backslash \mathbf{C} .
$$

We call any map $K$ as above, a $W$-feedback for the control system (2.1). When the dependence of $K$ on $W$ is clear, we simply call $K$ a feedback.

\subsection{Proof of the sample stabilizability with $\left(p_{0}, W\right)$-regulated cost}

The proof relies on Propositions 3.4, 3.5 and on Lemma 3.6 below.

Proposition 3.4. [19, Prop. 3.5] Assume (H0). Let $W$ be a $p_{0}-M R F$ with $p_{0} \geq 0$, define $N$ accordingly to Proposition 3.2, and let $K$ be a $W$-feedback. Moreover, let $\varepsilon, \hat{\mu}, \sigma$ verify $\varepsilon>0$ and $0<\hat{\mu}<\sigma$. Then there exists some $\hat{\delta}=\hat{\delta}(\hat{\mu}, \sigma)>0$ such that, for every partition $\pi=\left(t^{j}\right)$ of $[0,+\infty)$ with $\operatorname{diam}(\pi) \leq \hat{\delta}$ and for each $z \in \mathbb{R}^{n} \backslash \mathbf{C}$ satisfying $W(z) \in(\hat{\mu}, \sigma]$, any $\pi$-sampling trajectory-control pair $(x, u)$ of

$$
\dot{x}=f(x, u), \quad x(0)=z,
$$

associated to the feedback $K$ is defined on $[0, \hat{t})$ and enjoys the following properties: 
(i) $\hat{t}:=T_{x}^{\hat{\mu}}<+\infty$, where

$$
T_{x}^{\hat{\mu}}:=\inf \{t \geq 0: W(x(t)) \leq \hat{\mu}\} ;
$$

(ii) for every $t \in[0, \hat{t})$ and $j \geq 1$ such that $t \in\left[t^{j-1}, t^{j}\right)$,

$W(x(t))-W\left(x\left(t^{j-1}\right)\right)+p_{0} \int_{t^{j-1}}^{t} l(x(\tau), u(\tau)) d \tau \leq-\frac{\gamma\left(W\left(x\left(t^{j-1}\right)\right)\right)}{\varepsilon+1}\left(t-t^{j-1}\right)$.

Proposition 3.4 describes the behavior of any sampling trajectory-control pair $(x, u)$ with sampling time not greater than $\hat{\delta}$ just until its first exit-time $\hat{t}$ from the set $\left\{x \in \overline{\mathbb{R}^{n} \backslash \mathbf{C}}: W(x)>\hat{\mu}\right\}$. In [19] this was enough to derive global asymptotic controllability. Global asymptotic stabilizability, instead, requires also that, loosely speaking, any $x$ is defined in $[0,+\infty)$ and stays in the sublevel set $\left\{x \in \overline{\mathbb{R}^{n} \backslash \mathbf{C}}: W(x) \leq \hat{\mu}\right\}$ for every $t \geq \bar{t}$, for some $\bar{t}=\bar{t}(\hat{\mu}, \sigma)$. This is the content of the next proposition, which can be seen as an extension of [13, Lemma IV.2] to the setting considered here.

Proposition 3.5. Assume $(\mathrm{H} 0)$ and let $W$ be a $p_{0}-M R F$ with $p_{0} \geq 0$. Using the same notation of Proposition 3.4, set

$$
\bar{\delta}=\bar{\delta}(\hat{\mu}, \sigma):=\min \left\{\hat{\delta}\left(\frac{\hat{\mu}}{4}, 2 \sigma\right), \frac{\hat{\mu}}{4 L m}\right\},
$$

where $L$ is the Lipschitz constant of $W$ in $W^{-1}([\hat{\mu} / 4,2 \sigma])$ and

$$
m:=\sup _{W^{-1}((0,2 \sigma]) \times U}|(f, l)| .
$$

Then for every partition $\pi=\left(t^{j}\right)$ of $[0,+\infty)$ with $\operatorname{diam}(\pi) \leq \bar{\delta}$ and for each $z \in \mathbb{R}^{n} \backslash \mathbf{C}$ satisfying $W(z) \in(\hat{\mu}, \sigma]$, any $\pi$-sampling trajectory $x$ of $(3.5)$ is defined in $[0,+\infty)^{4}$ and verifies

$$
x(t) \in W^{-1}([0, \hat{\mu}]) \quad \forall t \geq \bar{t},
$$

where $\bar{t}:=T_{x}^{\hat{\mu} / 4}<+\infty$.

Proof. Fix a partition $\pi=\left(t^{j}\right)$ of $[0,+\infty)$ of diameter not greater than $\bar{\delta}$ and an initial datum $z \in W^{-1}((\hat{\mu}, \sigma])$. By Proposition 3.4 with $\hat{\mu} / 4$ in place of $\hat{\mu}$, any $\pi$-sampling solution $x$ is defined at least up to $\bar{t}:=T_{x}^{\hat{\mu} / 4}<+\infty$ and $W(x([0, \bar{t}]) \subset[\hat{\mu} / 4, W(z)], W(x(\bar{t}))=\hat{\mu} / 4$. Moreover, if $\bar{n}:=\max \{j \in \mathbb{N}:$ $\left.t^{j} \leq \bar{t}\right\}$, then we have

$$
x(t) \in W^{-1}\left(\left[\hat{\mu} / 4, W\left(t^{\bar{n}-1}\right)\right]\right) \subseteq W^{-1}([\hat{\mu} / 4,3 \hat{\mu} / 4]) \quad \forall t \in\left[t^{\bar{n}-1}, t^{\bar{n}}\right] .
$$

where, to deal with the case $\bar{n}=0$, we set $t^{-1}:=t^{0}=0$. The last inclusion follows by the definition of $\bar{\delta}$, which implies

$$
W\left(x\left(t^{\bar{n}}\right)\right)-W(x(\bar{t})) \leq L\left|x\left(t^{\bar{n}}\right)-x(\bar{t})\right| \leq L m \bar{\delta} \leq \frac{\hat{\mu}}{4},
$$

\footnotetext{
${ }^{4}$ When $T_{x}<+\infty$, we always mean that $x:\left[0, T_{x}\right) \rightarrow \mathbb{R}^{n} \backslash \mathbf{C}$ is extended to $[0,+\infty)$ as described in Definition 2.1.
} 
so that $W\left(x\left(t^{\bar{n}}\right)\right) \leq \hat{\mu} / 2$ and, arguing similarly, $W\left(x\left(t^{\bar{n}-1}\right)\right) \leq 3 \hat{\mu} / 4$.

We use (3.11) as base to inductively prove that any $\pi$-sampling solution $x$ of (3.5) either is defined on $[0,+\infty)$ and verifies (3.10) in the stronger form

$$
x(t) \in W^{-1}((0, \hat{\mu}]) \quad \forall t \geq \bar{t},
$$

or $x$ has finite blow-up time coinciding with the first time $T_{x}$ such that $\lim _{t \rightarrow T_{x}^{-}} \mathbf{d}(x(t))=0$ : in this case, since $|\dot{x}|$ is bounded by $m, x$ can be continuously extended to $[0,+\infty)$ and this extension verifies (3.10).

Fix $j \geq \bar{n}$ and assume by induction that an arbitrary $\pi$-sampling trajectory $x$, eventually extended accordingly to Definition 2.1, is defined up to time $t^{j-1}$ and verifies $x\left(\left[0, t^{j-1}\right]\right) \subseteq W^{-1}([0, \hat{\mu}])$. We have to show that $x$ is defined on $\left[t^{j-1}, t^{j}\right]$ and verifies

$$
x(t) \in W^{-1}([0, \hat{\mu}]) \quad \forall t \in\left[t^{j-1}, t^{j}\right] .
$$

If $W\left(x\left(t^{j-1}\right)\right)=0, x$ is constant on $\left[t^{j-1}, t^{j}\right]$ and (3.12) is obviously satisfied. When $0<W\left(x\left(t^{j-1}\right)\right) \leq \hat{\mu}$, we distinguish the following situations:

CASE 1 . $W\left(x\left(t^{j-1}\right)\right) \geq \hat{\mu} / 2$. Then by Proposition 3.4 (choosing in particular $z=x\left(t^{j-1}\right)$ and the partition $\left.\pi_{j}:=\left(t^{k+j-1}-t^{j-1}\right)_{k}\right)$ we deduce that any $\pi$-sampling trajectory with value $W\left(x\left(t^{j-1}\right)\right) \geq \hat{\mu} / 2$ is defined on the whole interval $\left[t^{j-1}, t^{j}\right]$ and verifies $0 \leq W(x(t))-W\left(x\left(t^{j}\right)\right) \leq L m \bar{\delta} \leq \hat{\mu} / 4$ for all $t \in\left[t^{j-1}, t^{j}\right]$, so that $x\left(\left[t^{j-1}, t^{j}\right]\right) \subset W^{-1}([\hat{\mu} / 4, \hat{\mu}])$ and this implies (3.12).

CASE 2. $W\left(x\left(t^{j-1}\right)\right)<\hat{\mu} / 2$. Any $\pi$-sampling solution $x$ of (3.5) with this property can be defined on a maximal interval $\left[t^{j-1}, \tilde{t}\right)$. Assume first that $\tilde{t}>t^{j}$, so that $x$ is defined for all $t \in\left[t^{j-1}, t^{j}\right]$ and suppose by contradiction

$$
x\left(\left[t^{j-1}, t^{j}\right]\right) \nsubseteq W^{-1}([0, \hat{\mu}]) .
$$

Then there exist $t^{i-1}<\underline{t}^{j}<\bar{t}^{j} \leq t^{j}$ such that

$$
W\left(x\left(\underline{t}^{j}\right)\right)=\hat{\mu} / 2, \quad W\left(x\left(\bar{t}^{j}\right)\right)=\hat{\mu}, \quad x\left(\left[\underline{t}^{j}, \bar{t}^{j}\right]\right) \subseteq W^{-1}([\hat{\mu} / 2, \hat{\mu}]) .
$$

This yields the required contradiction, since we have

$$
\hat{\mu} / 2=W\left(x\left(\bar{t}^{j}\right)\right)-W\left(x\left(\underline{t}^{j}\right)\right) \leq L m \bar{\delta} \leq \hat{\mu} / 4 .
$$

Therefore $x$ verifies $(3.12)$.

Let us now assume $\tilde{t} \leq t^{j}$. By standard properties of the ODEs, the blow-up time $\tilde{t}$ verifies either $\lim _{t \rightarrow \tilde{t}^{-}}|x(t)|=+\infty$ or $\tilde{t}=T_{x}$. Notice that if we had

$$
x\left(\left[t^{j-1}, \tilde{t}\right)\right) \nsubseteq W^{-1}([0, \hat{\mu}]),
$$

we could find $t^{i-1}<\underline{t}^{j}<\bar{t}^{j}<\tilde{t}$ and obtain a contradiction arguing as above. Hence $x\left(\left[t^{j-1}, \tilde{t}\right)\right) \subseteq W^{-1}([0, \hat{\mu}])$ and $\tilde{t}=T_{x}$ necessarily, since the set $W^{-1}([0, \hat{\mu}])$ is compact. By the boundedness of $f$ on $W^{-1}((0, \hat{\mu}]) \times U$ this implies that $\exists \lim _{t \rightarrow \tilde{t}} x(t)=\bar{z} \in \partial \mathbf{C}$ and the extension of $x$ to $\left[t^{j-1}, t^{j}\right]$ given by $x(t)=\bar{z}$ for all $t \in\left[\tilde{t}, t^{j}\right]$ verifies (3.12). The proof is thus concluded.

Finally, let us relate the level sets of a $p_{0}-\mathrm{MRF} W$ with the ones of the distance function $\mathbf{d}$ using the following general result. 
Lemma 3.6. Let $W, W_{1}: \overline{\mathbb{R}^{n} \backslash \mathbf{C}} \rightarrow[0,+\infty)$ be continuous functions, and let us assume that $W$ and $W_{1}$ are positive definite and proper on $\mathbb{R}^{n} \backslash \mathbf{C}$. Then the functions $\bar{g}, \underline{g}:(0,+\infty) \rightarrow(0,+\infty)$ given by

$$
\begin{aligned}
& \underline{g}(r)=\underline{g}_{W, W_{1}}(r):=\sup \left\{\alpha>0: \quad\{\tilde{z}: W(\tilde{z}) \leq \alpha\} \subseteq\left\{\tilde{z}: W_{1}(\tilde{z})<r\right\}\right\}, \\
& \bar{g}(r)=\bar{g}_{W, W_{1}}(r):=\inf \left\{\alpha>0: \quad\{\tilde{z}: W(\tilde{z}) \leq \alpha\} \supseteq\left\{\tilde{z}: W_{1}(\tilde{z}) \leq r\right\}\right\},
\end{aligned}
$$

are well-defined, increasing and there exist the limits

$$
\lim _{r \rightarrow 0^{+}} \bar{g}(r)=\lim _{r \rightarrow 0^{+}} \underline{g}(r)=0, \quad \lim _{r \rightarrow+\infty} \bar{g}(r)=\lim _{r \rightarrow+\infty} \underline{g}(r)=+\infty .
$$

Moreover, one has

$$
\underline{g}\left(W_{1}(x)\right) \leq W(x) \leq \bar{g}\left(W_{1}(x)\right) \quad \forall x \in \mathbb{R}^{n} \backslash \mathbf{C} .
$$

Proof. For every $\alpha>0$, let us introduce the sets $\mathcal{S}_{\alpha}:=\{\tilde{z}: W(\tilde{z}) \leq \alpha\}$, $\mathcal{S}_{\alpha}^{1}:=\left\{\tilde{z}: W_{1}(\tilde{z}) \leq \alpha\right\}$, and $\mathcal{S}_{\alpha}^{1<}:=\left\{\tilde{z}: W_{1}(\tilde{z})<\alpha\right\}$. By the hypotheses on $W$ and $W_{1}$ it follows that $\left(\mathcal{S}_{\alpha}\right)_{\alpha>0},\left(\mathcal{S}_{\alpha}^{1}\right)_{\alpha>0}$, and $\left(\mathcal{S}_{\alpha}^{1<}\right)_{\alpha>0}$ are strictly increasing families of nonempty, bounded sets verifying

$$
\begin{aligned}
& \lim _{\alpha \rightarrow 0^{+}} \mathcal{S}_{\alpha}=\lim _{\alpha \rightarrow 0^{+}} \mathcal{S}_{\alpha}^{1}=\lim _{\alpha \rightarrow 0^{+}} \mathcal{S}_{\alpha}^{1<}=\mathbf{C}, \\
& \lim _{\alpha \rightarrow+\infty} \mathcal{S}_{\alpha}=\lim _{\alpha \rightarrow+\infty} \mathcal{S}_{\alpha}^{1}=\lim _{\alpha \rightarrow+\infty} \mathcal{S}_{\alpha}^{1<}=\mathbb{R}^{n} .
\end{aligned}
$$

Then for any $r>0$ there exist $\bar{\alpha}, \bar{\alpha}_{1}>0$ such that $\mathcal{S}_{\alpha} \subset \mathcal{S}_{r}^{1<}$ for all $\alpha \leq \bar{\alpha}$ and $\mathcal{S}_{\alpha} \supset \mathcal{S}_{r}^{1}$ for all $\alpha \geq \bar{\alpha}_{1}$, so that $\bar{g}(r)$ and $\underline{g}(r)$ turn out to be well-defined. Moreover, $r \mapsto \bar{g}(r), g(r)$ are clearly increasing and verify the limits (3.14).

In order to prove the inequalities in (3.15), given $x \in \mathbb{R}^{n} \backslash \mathbf{C}$, let us set $r:=W_{1}(x), \alpha:=W(x), \underline{\alpha}:=\underline{g}\left(W_{1}(x)\right)$, and $\bar{\alpha}:=\bar{g}\left(W_{1}(x)\right)$. Arguing by contradiction, let us first assume that $\underline{g}\left(W_{1}(x)\right)>W(x)$, namely

$$
\underline{\alpha}>\alpha \text {. }
$$

By the definition of $\underline{\alpha}$, (3.16) would imply that $\mathcal{S}_{\alpha} \subseteq \mathcal{S}_{r}^{1<}$. This is impossible, since $x \in \mathcal{S}_{\alpha}$ but $x \notin \mathcal{S}_{r}^{1<}$, because $\alpha=W(x) \leq \alpha$ while $r=W_{1}(x) \nless r$. Similarly, if we suppose that $W(x)>\bar{g}\left(W_{1}(x)\right)$, namely

$$
\bar{\alpha}<\alpha,
$$

by the definition of $\bar{\alpha}$ we get that, for every $\alpha^{\prime} \in(\bar{\alpha}, \alpha)$, one should have $\mathcal{S}_{r}^{1} \subseteq \mathcal{S}_{\alpha^{\prime}}$. But this contradicts the fact that $x \in \mathcal{S}_{r}^{1}$, since $r=W_{1}(x) \leq r$, while $x \notin \mathcal{S}_{\alpha^{\prime}}$, being $\alpha=W(x) \not \leq \alpha^{\prime}$.

We are now ready to show that, given a $p_{0}$-MRF $W$ with $p_{0}>0$, the control system (2.1) is sample stabilizable to $\mathbf{C}$ with $\left(p_{0}, W\right)$-regulated cost. For any pair $r, R>0$ with $r<R$, let us set

$$
\begin{aligned}
& \hat{\mu}(r):=\underline{g}_{W, \mathbf{d}}(r)=\sup \left\{\mu>0: \quad\{\tilde{z}: W(\tilde{z}) \leq \mu\} \subseteq \stackrel{\circ}{B}_{r}(\mathbf{C})\right\}, \\
& \sigma(R):=\bar{g}_{W, \mathbf{d}}(R)=\inf \left\{\sigma>0: \quad\{\tilde{z}: W(\tilde{z}) \leq \sigma\} \supseteq B_{R}(\mathbf{C})\right\} .
\end{aligned}
$$


By Lemma 3.6, if $r<\mathbf{d}(\tilde{z}) \leq R$, then $\tilde{z} \in W^{-1}((\hat{\mu}(r), \sigma(R)])$ and the values $\hat{\mu}(r), \sigma(R)$ are finite and verify $0<\hat{\mu}(r)<\sigma(R)$. Let us choose

$$
\delta=\delta(r, R):=\bar{\delta}(\hat{\mu}(r), \sigma(R)),
$$

where $\bar{\delta}(\hat{\mu}, \sigma)$ is defined by (3.8). Fixed $\varepsilon>0$, for instance, $\varepsilon=1$, by Propositions $3.4,3.5$ it follows that for every partition $\pi=\left(t^{j}\right)$ of $[0,+\infty)$ with $\operatorname{diam}(\pi) \leq \delta$ and for every initial state $z \in \mathbb{R}^{n} \backslash \mathbf{C}$ such that $\mathbf{d}(z) \leq R$, any $\pi$-sampling trajectory-control pair $(x, u)$ of $(2.1)$ with $x(0)=z$ has $x$ defined in $[0,+\infty)$ and verifies:

(i) $\bar{t}:=T_{x}^{\hat{\mu}(r) / 4}<+\infty$;

(ii) for every $t \in[0, \bar{t})$ and $j \geq 1$ such that $t \in\left[t^{j-1}, t^{j}\right)$,

$W(x(t))-W\left(x\left(t^{j-1}\right)\right)+p_{0} \int_{t^{j-1}}^{t} l(x(\tau), u(\tau)) d \tau \leq-\frac{\gamma\left(W\left(x\left(t^{j-1}\right)\right)\right)}{2}\left(t-t^{j-1}\right) ;$

(iii) for every $t \geq \bar{t}, W(x(t)) \leq \hat{\mu}(r)$, which implies that $\mathbf{d}(x(t)) \leq r$.

The time $\bar{t}$ might be zero when $\mathbf{d}(z) \leq r$. Of course, condition (ii) is significant only if $\bar{t}>0$.

Observing that (3.20) implies

$$
W(x(t))-W(z) \leq-\frac{\gamma\left(W\left(x\left(t^{j-1}\right)\right)\right)}{2} t \quad \forall t \in[0, \bar{t}),
$$

the construction of a $\mathcal{K} \mathcal{L}$ function $\beta$ such that

$$
\mathbf{d}(y(t)) \leq \beta(\mathbf{d}(z), t) \quad \forall t \in[0, \bar{t})
$$

can be obtained arguing as in [19, p.600], hence we omit it. Together with (iii), this yields that

$$
\mathbf{d}(x(t)) \leq \max \{\beta(\mathbf{d}(z), t), r\} \quad \forall t \geq 0 .
$$

Moreover, when $\bar{t}>0$ by summing up $j$ from 0 to the last index $\tilde{n}$ such that $t^{\tilde{n}}<\bar{t}$, from (ii) it follows that

$$
W(x(\bar{t}))-W(z)+p_{0} \int_{0}^{\bar{t}} l(x(\tau), u(\tau)) d \tau \leq-\frac{\gamma\left(W\left(x\left(t^{\tilde{n}}\right)\right)\right)}{2} \bar{t} .
$$

Hence

$$
\int_{0}^{\bar{t}} l(x(\tau), u(\tau)) d \tau \leq \frac{W(z)}{p_{0}}
$$

and this concludes the proof since

$$
\bar{t} \geq \bar{T}_{x}^{r}=\inf \{t>0: \mathbf{d}(x(\tau)) \leq r \quad \forall \tau \geq t\} .
$$


Remark 3.7. When $\mathbf{d}(z)>r$, the time $\bar{T}_{x}^{r}$, after which any $(r, R)$ stable $\pi$ sampling trajectory $x$ starting from $z$ remains definitively in $B_{r}(\mathbf{C})$, is uniformly bounded by a positive constant. Precisely, using the above notations, by the previous proof one can easily deduce the following upper bound

$$
\bar{T}_{x}^{r} \leq \bar{t} \leq \frac{2(W(z)-W(x(\bar{t})))}{\gamma(W(x(\bar{t})))}=\frac{2\left(W(z)-\frac{\hat{\mu}(r)}{4}\right)}{\gamma(\hat{\mu}(r) / 4)} .
$$

\subsection{Proof of the Euler stabilizability with $\left(p_{0}, W\right)$-regulated cost}

Let us start with some preliminary results. In the sequel we make use of all the notations introduced in the previous subsection.

The following lemma establishes a uniform lower bound for the time needed to admissible trajectories starting from the same point $z$ and approaching the target, to reach an $\varepsilon$-neighborhood of the target.

Lemma 3.8. Assume (H0). Given $R>0$, let us set

$$
\tilde{M}(R):=\sup \left\{|f(x, u)|: \quad x \in B_{R}(\mathbf{C}) \backslash \mathbf{C}, \quad u \in U\right\} .
$$

Then for any $z \in \mathbb{R}^{n} \backslash \mathbf{C}$ such that $\mathbf{d}(z) \leq R$ and $\varepsilon \in(0, \mathbf{d}(z))$, setting

$$
T_{\varepsilon}:=\frac{\mathbf{d}(z)-\varepsilon}{\tilde{M}(R)}>0,
$$

every admissible trajectory-control pair $(x, u) \in \mathcal{A}_{f}(z)$ with $T_{x} \leq+\infty$ and such that $\lim _{t \rightarrow T_{x}^{-}} \mathbf{d}(x(t))=0$, verifies

$$
\mathbf{d}(x(t)) \geq \varepsilon \quad \forall t \in\left[0, T_{\varepsilon}\right] .
$$

As a consequence, $T_{x} \geq \frac{\mathbf{d}(z)}{\tilde{M}(R)}$.

Proof. Given $(x, u) \in \mathcal{A}_{f}(z)$ as above, let us set $\bar{\tau}:=\sup \{t \geq 0: \mathbf{d}(x(t)) \geq$ $\mathbf{d}(z)\}$. The time $\bar{\tau}$ is clearly finite and defining $\tilde{T}_{x}^{\varepsilon}:=\inf \{t>\bar{\tau}: \mathbf{d}(x(t)) \leq \varepsilon\}$, one trivially has $0 \leq \bar{\tau}<\tilde{T}_{x}^{\varepsilon}$ and $x\left(\left[\bar{\tau}, \tilde{T}_{x}^{\varepsilon}\right]\right) \subseteq \overline{B_{R}(\mathbf{C})} \backslash \mathbf{C}$. If $\bar{z}^{\varepsilon} \in \partial \mathbf{C}$ verifies

$$
\varepsilon=\mathbf{d}\left(x\left(\tilde{T}_{x}^{\varepsilon}\right)\right)=\left|x\left(\tilde{T}_{x}^{\varepsilon}\right)-\bar{z}^{\varepsilon}\right|,
$$

then the uniform bound (3.26) is a consequence of the following inequalities $\mathbf{d}(z)=\mathbf{d}(x(\bar{\tau})) \leq\left|x(\bar{\tau})-\bar{z}^{\varepsilon}\right| \leq\left|x(\bar{\tau})-x\left(\tilde{T}_{x}^{\varepsilon}\right)\right|+\left|x\left(\tilde{T}_{x}^{\varepsilon}\right)-\bar{z}^{\varepsilon}\right| \leq \tilde{M}(R) \tilde{T}_{x}^{\varepsilon}+\varepsilon$, implying that $\tilde{T}_{x}^{\varepsilon} \geq \frac{\mathbf{d}(z)-\varepsilon}{\tilde{M}(R)}=T_{\varepsilon}$. Indeed, $\mathbf{d}(x(t)) \geq \mathbf{d}(z)>\varepsilon$ for all $t \in[0, \bar{\tau}]$ and the definition of $\tilde{T}_{x}^{\varepsilon}$ implies that $\mathbf{d}(x(t))>\varepsilon$ for all $\left.\left.t \in\right] \bar{\tau}, \tilde{T}_{x}^{\varepsilon}\right]$, so that $\mathbf{d}(x(t)) \geq \varepsilon$ for all $t \in\left[0, T_{\varepsilon}\right]$. By the arbitrariness of $\varepsilon>0$, this implies that $T_{x} \geq \mathbf{d}(z) / \tilde{M}(R)$.

Next result allows us to determine, given a $p_{0}-\mathrm{MRF} W$, a positive constant $R$ and a sampling time $\delta>0$ small enough, a radius $r<R$ such that any $\pi$-sampling trajectory-control pair for (3.5) with initial point $z$ verifying $\mathbf{d}(z) \leq R$ and with $\operatorname{diam}(\pi)=\delta$ is $(r, R)$-stable. 
Lemma 3.9. Assume (H0). Let $W$ be a $p_{0}-M R F$ with $p_{0} \geq 0$ and for any pair $r, R>0$ with $r<R$, let $\delta=\delta(r, R)$ be defined accordingly to (3.19). Then, for every fixed $R>0, \delta(\cdot, R)$ is positive and increasing and

$$
\lim _{r \rightarrow 0^{+}} \delta(r, R)=0, \quad \delta(R):=\lim _{r \rightarrow R^{-}} \delta(r, R)<+\infty .
$$

Proof. By Sect. 3.1, we have that

$$
\delta(r, R)=\bar{\delta}(\mu(r), \sigma(R)),
$$

where $\bar{\delta}$ is defined as in (3.8), in Proposition 3.5. Since the map $r \mapsto \hat{\mu}(r)$ is increasing, $\hat{\mu}(r)$ vanishes as $r \rightarrow 0^{+}$and $\hat{\mu}(r)$ is bounded by $\sigma(R)$ as $r \rightarrow R^{-}$, to conclude it suffices to show that for every $\sigma>0$ the map $\hat{\mu} \mapsto \bar{\delta}(\hat{\mu}, \sigma)$ (a) is increasing in $(0, \sigma)$, (b) vanishes in 0 and (c) is bounded as $\hat{\mu}$ tends to $\hat{\mu}(R)$. Let $L(\hat{\mu}, \sigma)$ be the Lipschitz constant of $W$ on $W^{-1}([\hat{\mu}, 2 \sigma])$, let $m=m(\sigma)$ be as in (3.9) and recall from (3.8) the following definition

$$
\bar{\delta}(\hat{\mu}, \sigma)=\min \left\{\hat{\delta}\left(\frac{\hat{\mu}}{4}, 2 \sigma\right), \frac{\hat{\mu}}{4 L(\hat{\mu}, \sigma) m}\right\} .
$$

We note that $\mu \hat{\mapsto} L(\hat{\mu}, \sigma)$ is decreasing in $(0, \sigma)$ : this implies at once conditions (b) and (c) and the fact that, for every $\sigma>0$, the map $\hat{\mu} \mapsto \hat{\mu} / 4 L(\hat{\mu}, \sigma) m$ is increasing. To conclude it is left to show that, for every $\sigma>0$, the map $\hat{\mu} \mapsto \hat{\delta}(\hat{\mu}, \sigma)$ is increasing in $(0, \sigma)$. This monotonicity that can be easily derived arguing as above, by the definitions of the constants in the proof of [19, Proposition 3.5], hence we omit to prove it.

Owing to Lemma 3.9, given a $p_{0}-\mathrm{MRF} W$ and a positive constant $R$, we can assume without loss of generality that $\delta(\cdot, R)$ defined as above is strictly increasing and continuous. Therefore, for any $R>0$ we can define the inverse of the map $r \mapsto \delta(r):=\delta(r, R)$, given by

$$
\delta \mapsto r(\delta) \quad \forall \delta \in[0, \delta(R)],
$$

which is continuous, strictly increasing and such that $r(0)=0$ and $r(\delta(R))=$ $R$. As an immediate consequence, by the sample stabilizability of $(2.1)$ with $\left(p_{0}, W\right)$-regulated cost we get the following result.

Lemma 3.10. Assume $(\mathrm{H} 0)$ and let $W$ be a $p_{0}-M R F$ with $p_{0} \geq 0$. Then there exists a function $\beta \in \mathcal{K} \mathcal{L}$ such that, for each pair $R>0$ and $\delta \in(0, \delta(R))$, for every partition $\pi$ of $[0,+\infty)$ with $\operatorname{diam}(\pi)=\delta$ and for any initial state $z \in \mathbb{R}^{n} \backslash \mathbf{C}$ such that $\mathbf{d}(z) \leq R$, any $\pi$-sampling trajectory-control pair $(x, u)$ of (2.1) from $z$ is defined in $[0,+\infty)$ and verifies:

$$
\mathbf{d}(x(t)) \leq \max \{\beta(R, t), r(\delta)\} \quad \forall t \geq 0 .
$$

Moreover, if $p_{0}>0$,

$$
x^{0}\left(\bar{T}_{x}^{r(\delta)}\right)=\int_{0}^{\bar{T}_{x}^{r(\delta)}} l(x(\tau), u(\tau)) d \tau \leq \frac{W(z)}{p_{0}},
$$

where $\bar{T}_{x}^{r(\delta)}=\inf \{t>0: \mathbf{d}(x(\tau)) \leq r(\delta) \forall \tau \geq t\}$, as in (2.6). 
Remark 3.11. When $f$ and $l$ verify hypothesis (H0) and system (2.1) is sample stabilizable to $\mathbf{C}$ with $\left(p_{0}, W\right)$-regulated cost, there always exist continuous Euler solutions to (2.1). Indeed, for any $z$ with $0<\mathbf{d}(z) \leq R$ and any sequence $\left(x_{i}, u_{i}\right)$ of $\pi_{i}$-sampling trajectory-control pairs of $(2.1)$ with $x(0)=z$, $\delta_{i}:=\operatorname{diam}\left(\pi_{i}\right) \rightarrow 0$ as $i \rightarrow+\infty$, and associated costs $x_{i}^{0}$, it turns out that $\left(x_{i}^{0}, x_{i}\right)$ is equi-Lipschitz continuous on $[0,+\infty)$ with Lipschitz constant $m>0$. Hence the existence of continuous, actually $m$-Lipschitz continuous Euler solutions to (2.1) from $z$ with $m$-Lipschitz continuous Euler costs follows straightforwardly by Ascoli-Arzelá's Theorem.

We are now in position to prove that, if we assume (H0) and $W$ is a $p_{0}$-MRF with $p_{0}>0$, the feedback $K$ Euler-stabilizes the system (2.1) to $\mathbf{C}$ with $\left(p_{0}, W\right)$-regulated cost. Given $z \in \mathbb{R}^{n} \backslash \mathbf{C}$, let $\left(X^{0}, \mathcal{X}\right)$ be an Euler solution of (2.1) with initial condition $X(0)=z$. By definition there exist a sequence of partitions $\left(\pi_{i}\right)$ of $[0,+\infty)$ such that $\delta_{i}:=\operatorname{diam}\left(\pi_{i}\right) \rightarrow 0$ as $i \rightarrow \infty$, a sequence of $\pi_{i}$-sampling trajectory-control pairs $\left(x_{i}, u_{i}\right)$ for $(2.1)$ with $x_{i}(0)=z$ for each $i$, and associated costs $x_{i}^{0}$, satisfying

$$
\left(x_{i}^{0}, x_{i}\right) \rightarrow\left(X^{0}, X\right) \text { locally uniformly on }[0,+\infty) .
$$

Set $R:=\mathbf{d}(z)$ and let $\beta, \delta(R)$ and $r:[0, \delta(R)] \rightarrow[0, R]$ be as in Lemma 3.10. Since $\delta_{i} \rightarrow 0$, we can assume without loss of generality that $\delta_{i}<\delta(R)$ for all $i$. Hence Lemma 3.10 implies that, for every $i$,

$$
\mathbf{d}\left(x_{i}(t)\right) \leq \max \left\{\beta(\mathbf{d}(z), t), r\left(\delta_{i}\right)\right\} \quad \forall t \geq 0
$$

and

$$
x_{i}^{0}(t) \leq \frac{W(z)}{p_{0}} \quad \forall t \in\left[0, \bar{T}_{x_{i}}^{r\left(\delta_{i}\right)}\right] .
$$

As $i \rightarrow \infty$, we have that $\delta_{i} \rightarrow 0$ and consequently $r\left(\delta_{i}\right) \rightarrow 0$. Then by (3.30) and (3.31) we obtain that

$$
\mathbf{d}(X(t)) \leq \beta(\mathbf{d}(z), t) \quad \forall t \geq 0 .
$$

Hence $\lim _{t \rightarrow+\infty} \mathbf{d}(X(t))=0$ and there exists

$$
T_{X}:=\inf \left\{\tau \geq 0: \quad \lim _{t \rightarrow \tau^{-}} \mathbf{d}(X(t))=0\right\} \leq+\infty .
$$

To conclude the proof it remains only to show that

$$
\lim _{t \rightarrow T_{x}^{-}} X^{0}(t) \leq \frac{W(z)}{p_{0}},
$$

where the limit is well defined, since $X^{0}$, pointwise limit of monotone nondecreasing functions, is monotone nondecreasing. Passing eventually to a subsequence, we set $\bar{T}:=\lim _{i} \bar{T}_{x_{i}}^{r\left(\delta_{i}\right)}$. In view of Lemma $3.8, \bar{T}$ satisfies

$$
\bar{T} \geq \frac{\mathbf{d}(z)}{m}>0 .
$$


Then for any $t \in[0, \bar{T})$ one has $\bar{T}_{x_{i}}^{r\left(\delta_{i}\right)}>t$ for all $i$ sufficiently large and, taking the limit as $i \rightarrow \infty$ in (3.32), by (3.30) it follows that

$$
X^{0}(t) \leq \frac{W(z)}{p_{0}} \quad \forall t \in[0, \bar{T}) .
$$

If $\bar{T}=+\infty$, this implies directly the thesis (3.34). If instead $\bar{T}<+\infty$, the definition of $\bar{T}_{x_{i}}^{r\left(\delta_{i}\right)}$ yields that

$$
\mathbf{d}\left(x_{i}\left(\bar{T}_{x_{i}}^{r\left(\delta_{i}\right)}\right)\right)=r\left(\delta_{i}\right) .
$$

Moreover, by the locally uniform convergence of $x_{i}$ to $\mathcal{X}$ and the $m$-Lipschitz continuity of $\mathcal{X}$, we get the following estimate

$$
\mathbf{d}(X(\bar{T})) \leq\left|X(\bar{T})-X\left(\bar{T}_{x_{i}}^{r\left(\delta_{i}\right)}\right)\right|+\left|X\left(\bar{T}_{x_{i}}^{r\left(\delta_{i}\right)}\right)-x_{i}\left(\bar{T}_{x_{i}}^{r\left(\delta_{i}\right)}\right)\right|+\mathbf{d}\left(x_{i}\left(\bar{T}_{x_{i}}^{r\left(\delta_{i}\right)}\right)\right),
$$

where the r.h.s. tends to zero as $i \rightarrow+\infty$. Thus we have in any case

$$
0<\frac{\mathbf{d}(z)}{m} \leq T_{X} \leq \bar{T},
$$

where the first inequality is again a consequence of the $m$-Lipschitz continuity of $X$. This concludes the proof, since (3.36) implies now the thesis (3.34).

\section{On the notion of $p_{0}$-minimum restraint function}

In Sect. 4.1 we obtain an equivalent formulation of the definition of $p_{0}$-MRF. Using this condition, in Sect. 4.2 we prove that, when the data $f$ and $l$ are locally Lipschitz continuous, the existence of a locally Lipschitz, not necessarily semiconcave, $p_{0}$-MRF $W$, still guarantees sample and Euler stabilizability of the control system (2.1) to $\mathbf{C}$ with $\left(p_{0}, W\right)$-regulated cost. Section 4.3 is devoted to extend these results to the original notion of $p_{0}-\mathrm{MRF}$ introduced in $[23]$.

\subsection{An equivalent notion of $p_{0}-\mathrm{MRF}$}

Proposition 4.1. Assume (H0). Let $W: \overline{\mathbb{R}^{n} \backslash \mathbf{C}} \rightarrow[0,+\infty)$, be a continuous function, which is positive definite, proper, and semiconcave on $\mathbb{R}^{n} \backslash \mathbf{C}$. Then $W$ is a $p_{0}-M R F$ for some $p_{0} \geq 0$ if and only if for any continuous function $W_{1}: \overline{\mathbb{R}^{n} \backslash \mathbf{C}} \rightarrow[0,+\infty)$, positive definite, and proper on $\mathbb{R}^{n} \backslash \mathbf{C}$, there is some continuous, strictly increasing function $\tilde{\gamma}:(0,+\infty) \rightarrow(0,+\infty)$ such that

$$
H\left(x, p_{0}, D^{*} W(x)\right) \leq-\tilde{\gamma}\left(W_{1}(x)\right) \quad \forall x \in \mathbb{R}^{n} \backslash \mathbf{C} .
$$

For instance, one can choose $W_{1}=\mathbf{d}$, distance function from $\mathbf{C}$.

Proposition 4.1 is a consequence of the following more general result, involving a locally Lipschitz, not necessarily semiconcave, function $W$.

Proposition 4.2. Assume $(\mathrm{H} 0)$ and let $W: \overline{\mathbb{R}^{n} \backslash \mathbf{C}} \rightarrow[0,+\infty)$ be a continuous map, which is locally Lipschitz, positive definite, and proper on $\mathbb{R}^{n} \backslash \mathbf{C}$. Given $p_{0} \geq 0$ and a set $\Omega \subseteq \mathbb{R}^{n} \backslash \mathbf{C}$, the following statements are equivalent: 
(i) $W$ verifies the decrease condition

$$
H\left(x, p_{0}, \partial_{P} W(x)\right) \leq-\gamma(W(x)) \quad \forall x \in \Omega,
$$

for some continuous, strictly increasing function $\gamma:(0,+\infty) \rightarrow(0,+\infty)$;

(ii) for any continuous function $W_{1}: \overline{\mathbb{R}^{n} \backslash \mathbf{C}} \rightarrow[0,+\infty)$ which is positive definite and proper on $\mathbb{R}^{n} \backslash \mathbf{C}$, there exists some continuous, strictly increasing function $\tilde{\gamma}:(0,+\infty) \rightarrow(0,+\infty)$ such that $W$ verifies

$$
H\left(x, p_{0}, \partial_{P} W(x)\right) \leq-\tilde{\gamma}\left(W_{1}(x)\right) \quad \forall x \in \Omega .
$$

Proof. Let us first prove that (i) $\Rightarrow$ (ii). Assume that $W$ verifies the decrease condition (4.1). Given an arbitrary continuous map $W_{1}$, positive definite and proper in $\mathbb{R}^{n} \backslash \mathbf{C}$, let $\tilde{\gamma}:(0,+\infty) \rightarrow(0,+\infty)$ be a continuous, strictly increasing approximation from below of the increasing map $r \mapsto \gamma \circ \underline{g}_{W, W_{1}}(r)$, where $\underline{g}_{W, W_{1}}$ is defined accordingly to Lemma 3.6. Then by (3.15), for any $x \in \mathbb{R}^{n} \backslash \mathbf{C}$, one has

$$
W(x) \geq \underline{g}_{W, W_{1}}\left(W_{1}(x)\right) \Longrightarrow \gamma(W(x)) \geq \gamma \circ \underline{g}_{W, W_{1}}\left(W_{1}(x)\right) \geq \tilde{\gamma}\left(W_{1}(x)\right),
$$

so that (4.1) implies (4.2) for such $\tilde{\gamma}$.

To prove that (ii) $\Rightarrow$ (i), it is enough to invert the roles of $W$ and $W_{1}$. Precisely, if (4.2) is verified for some $W, W_{1}$ and $\tilde{\gamma}$ as in the statement of the proposition, arguing as above one obtains that $W$ verifies (4.1) choosing as $\gamma:(0,+\infty) \rightarrow(0,+\infty)$ any continuous, strictly increasing approximation from below of the increasing map $r \mapsto \tilde{\gamma} \circ \underline{g}_{W_{1}, W}(r)$.

Proof of Proposition 4.1. The only non trivial fact in order to derive Proposition 4.1 from Proposition 4.2, is that (4.1) involves the proximal subdifferential $\partial_{P} W(x)$ at $x$ instead of the set of limiting gradients $D^{*} W(x)$ at $x$, considered in the decrease condition for a $p_{0}-\mathrm{MRF}$. However, when $W$ is locally Lipschitz continuous, condition (4.1) with $\Omega=\mathbb{R}^{n} \backslash \mathbf{C}$ implies readily the following inequality:

$$
H\left(x, p_{0}, \partial_{L} W(x)\right) \leq-\gamma(W(x)) \quad \forall x \in \mathbb{R}^{n} \backslash \mathbf{C},
$$

where $\partial_{L} W(x)$ denotes the limiting subdifferential at $x$. This concludes the proof, since a $p_{0}$-MRF $W$ is locally semiconcave and therefore $\partial_{L} W(x)=$ $D^{*} W(x)$ at any $x$.

\subsection{Lipschitz continuous $p_{0}-\mathrm{MRF}$}

Under the following hypothesis:

(H1) The sets $U \subset \mathbb{R}^{m}, \mathbf{C} \subset \mathbb{R}^{n}$ are closed and the boundary $\partial \mathbf{C}$ is compact. $f: \overline{\left(\mathbb{R}^{n} \backslash \mathbf{C}\right)} \times U \rightarrow \mathbb{R}^{n}, l: \overline{\left(\mathbb{R}^{n} \backslash \mathbf{C}\right)} \times U \rightarrow[0,+\infty)$ are continuous functions such that for every compact subset $\mathcal{K} \subset \overline{\mathbb{R}^{n} \backslash \mathbf{C}}$ there exist $M_{f}, M_{l}, L_{f}, L_{l}>0$ such that

$$
\left\{\begin{array}{l}
|f(x, u)| \leq M_{f}, \quad l(x, u) \leq M_{l} \quad \forall(x, u) \in \mathcal{K} \times U, \\
\left|f\left(x_{1}, u\right)-f\left(x_{2}, u\right)\right| \leq L_{f}\left|x_{1}-x_{2}\right|, \quad \forall\left(x_{1}, u\right),\left(x_{2}, u\right) \in \mathcal{K} \times U,
\end{array}\right.
$$

we obtain the main result of this section: 
Theorem 4.3. Assume (H1) and let $p_{0} \geq 0$. Let $W: \overline{\mathbb{R}^{n} \backslash \mathbf{C}} \rightarrow[0,+\infty)$ be a locally Lipschitz continuous map on $\overline{\mathbb{R}^{n} \backslash \mathbf{C}}$, such that $W$ is positive definite, and proper on $\mathbb{R}^{n} \backslash \mathbf{C}$, and verifies the decrease condition

$$
H\left(x, p_{0}, \partial_{P} W(x)\right) \leq-\tilde{\gamma}\left(W_{1}(x)\right) \quad \forall x \in \mathbb{R}^{n} \backslash \mathbf{C},
$$

for some continuous, strictly increasing function $\tilde{\gamma}:(0,+\infty) \rightarrow(0,+\infty)$ and some continuous function $W_{1}: \overline{\mathbb{R}^{n} \backslash \mathbf{C}} \rightarrow[0,+\infty)$, positive definite, and proper on $\mathbb{R}^{n} \backslash \mathbf{C}$. Then there exists a $\frac{p_{0}}{2}-M R F \bar{W}$, which also satisfies $\bar{W}(x) \leq W(x)$ for all $x \in \mathbb{R}^{n} \backslash \mathbf{C}$.

Theorem 4.3, whose proof is postponed to "Appendix A.1", generalizes the result on the existence of a semiconcave Control Lyapunov Function obtained in [28, sect. 5] to the present case, where the decrease condition involves also the Lagrangian $l$ and the target is not the origin, but an arbitrary closed set $\mathbf{C}$ with compact boundary.

Let us call a map $W$ as in Theorem 4.3 a Lipschitz continuous $p_{0}-M R F$. As an immediate consequence of Theorems 4.3 and 3.1, we have the following:

Corollary 4.4. Assume $(\mathrm{H} 1)$ and let $p_{0}>0$. Let $W: \overline{R^{n} \backslash \mathbf{C}} \rightarrow[0,+\infty)$ be a Lipschitz continuous $p_{0}-M R F$. Then there exists a locally bounded feedback $K: \mathbb{R}^{n} \backslash \mathbf{C} \rightarrow U$ that sample and Euler stabilizes system (1.1) to $\mathbf{C}$ with $\left(p_{0} / 2, W\right)$-regulated cost.

Note that, using the notation of Theorem 4.3, the feedback $K$ in Corollary 4.4 is actually a $\bar{W}$-feedback and the claim above relies on the inequality $\bar{W} \leq W$.

\subsection{Comparison with the original notion of $\boldsymbol{p}_{\mathbf{0}}-\mathrm{MRF}$}

Let us call $p_{0}$-OMRF the notion of $p_{0}$-MRF originally introduced in [23].

Definition 4.5. $\left(p_{0}\right.$-OMRF $)$ Let $W: \overline{\mathbb{R}^{n} \backslash \mathbf{C}} \rightarrow[0,+\infty)$ be a continuous function, and let us assume that $W$ is locally semiconcave, positive definite, and proper on $\mathbb{R}^{n} \backslash \mathbf{C}$. We say that $W$ is a $p_{0}-O M R F$ for some $p_{0} \geq 0$ if it verifies

$$
H\left(x, p_{0}, D^{*} W(x)\right)<0 \quad \forall x \in \mathbb{R}^{n} \backslash \mathbf{C} .
$$

A $p_{0}-\mathrm{MRF}$ is obviously a $p_{0}-\mathrm{OMRF}$, but the converse might be false. By [23] we have the following result.

Proposition 4.6. [23, Prop. 3.1] Assume that $W$ is a $p_{0}-O M R F$ with $p_{0} \geq 0$. Then for every $\sigma>0$ there exists a continuous, increasing map $\gamma_{\sigma}:(0, \sigma] \rightarrow$ $(0,+\infty)$ such that

$$
H\left(x, p_{0}, D^{*} W(x)\right)<-\gamma_{\sigma}(W(x)) \quad \forall x \in W^{-1}((0, \sigma]) .
$$

Proposition 4.6 clarifies the difference between the two notions: the existence of a $p_{0}$-OMRF implies that there exists a rate function $\gamma_{\sigma}$, which is in general, not global. In particular, $\gamma_{\sigma}$ can become smaller and smaller as $\sigma$ tends to $+\infty$. Consequently, also the feedback $K$ can be defined only given a $\sigma>0$, on $W^{-1}((0, \sigma])$. 
Remark 4.7. When a $p_{0}$-OMRF $W$ verifies condition (4.4) in the following stronger form

$$
\forall M>0: \quad \sup _{p \in D^{*} W(x)} H\left(x, p_{0}, p\right)<0 \quad \forall x \in \mathbb{R}^{n} \backslash \mathbf{C} \text { s.t. } \mathbf{d}(x) \geq M,
$$

it is not difficult to prove that, under the assumptions of Proposition 4.6, there exists a continuous, strictly increasing function $\gamma:(0,+\infty) \rightarrow(0,+\infty)$ independent of $\sigma$, such that (4.5) holds for all $x \in \mathbb{R}^{n} \backslash \mathbf{C}$ (see [23, Remark $3.1])$. In other words,

$$
\text { a } p_{0}-O M R F W \text { verifying (4.6) is actually a } p_{0}-M R F \text {. }
$$

The proof of Theorem 3.1 can be easily adapted to derive the following result.

Theorem 4.8. Assume that $f, l$ verify hypothesis $(\mathrm{H} 0)$ and let $W$ be a $p_{0^{-}}$ OMRF with $p_{0}>0$. Then for any $\sigma>0$ there exists a locally bounded feedback $K: W^{-1}((0, \sigma]) \rightarrow U$ that sample and Euler stabilizes system (2.1) to $\mathbf{C}$ with $\left(p_{0}, W\right)$-regulated cost for any initial point $z \in W^{-1}((0, \sigma])$.

As in the case of $p_{0}-\mathrm{MRF}$, when $f, l$ are locally Lipschitz continuous in $x$, we can replace the semiconcavity assumption in the definition of a $p_{0}$-OMRF with local Lipschitz continuity. Precisely, we establish what follows.

Theorem 4.9. Assume (H1) and let $p_{0} \geq 0$. Let $W: \overline{\mathbb{R}^{n} \backslash \mathbf{C}} \rightarrow[0,+\infty)$ be a locally Lipschitz continuous map on $\overline{\left(\mathbb{R}^{n} \backslash \mathbf{C}\right)}$, such that $W$ is positive definite, and proper on $\mathbb{R}^{n} \backslash \mathbf{C}$, and verifies the decrease condition

$$
H\left(x, p_{0}, \partial_{L} W(x)\right)<0 \quad \forall x \in \mathbb{R}^{n} \backslash \mathbf{C}
$$

Then there exists a $\frac{p_{0}}{2}$-OMRF $\bar{W}$ which also satisfies $\bar{W}(x) \leq W(x)$ for all $x \in \mathbb{R}^{n} \backslash \mathbf{C}$.

The proof of this theorem is sketched in "Appendix A.2".

Let us call a map $W$ as in Theorem 4.9 a Lipschitz continuous $p_{0}$-OMRF. Theorems 4.9 and 4.8 imply what follows:

Corollary 4.10. Assume $(\mathrm{H} 1)$ and let $p_{0}>0$. Let $W: \overline{\mathbb{R}^{n} \backslash \mathbf{C}} \rightarrow[0,+\infty)$ be a Lipschitz continuous $p_{0}-O M R F$. Then for any $\sigma>0$ there exists a locally bounded feedback $K: W^{-1}((0, \sigma]) \rightarrow U$ that sample and Euler stabilizes system (2.1) to $\mathbf{C}$ with $\left(p_{0} / 2, W\right)$-regulated cost for any initial point $z \in W^{-1}((0, \sigma])$.

\section{An example: stabilization of the non-holonomic integrator control system with regulated cost}

Let us illustrate the preceding theory through a classical example. Precisely, in the first part of this section we provide a $p_{0}$-MRF $W_{1}$ for the non-holonomic integrator control system associated to a Lagrangian $l$, that verifies a suitable growth condition (see (5.3) below). In view of Theorem 3.1, this implies the existence of a possibly discontinuous feedback $K$ that sample and Euler stabilizes the non-holonomic integrator to the origin with the cost bounded above 
by $W_{1} / p_{0}$. Furthermore, we show how weakening the requirements on the $p_{0^{-}}$ MRF (by replacing semiconcavity with Lipschitz continuity) may be crucial for the effective construction of a $p_{0}-\mathrm{MRF}$. In particular, for any bounded Lagrangian $l$ which cannot satisfy assumption (5.3) below for any $C>0$ (as, for instance, in the case of the minimum time problem, where $l \equiv 1$ ), we provide a less regular, Lipschitz continuous but not semiconcave $p_{0}$-MRF $W_{2}$. In this case, the sample and Euler stabilizability of the control system with $\left(p_{0} / 2, W_{2}\right)$-regulated cost is guaranteed by Corollary 4.4 .

Set $U:=\left\{u=\left(u_{1}, u_{2}\right) \in \mathbb{R}^{2}: u_{1}^{2}+u_{2}^{2} \leq 1\right\}, \mathbf{C}:=\{0\} \subset \mathbb{R}^{3}$ and consider the non-holonomic integrator control system:

$$
\left\{\begin{array}{l}
\dot{x}_{1}=u_{1} \\
\dot{x}_{2}=u_{2} \\
\dot{x}_{3}=x_{1} u_{2}-x_{2} u_{1}, \quad u(t)=\left(u_{1}, u_{2}\right)(t) \in U .
\end{array}\right.
$$

Given a continuous Lagrangian $l(x, u) \geq 0$, let us associate to (5.1) the cost

$$
\int_{0}^{T_{x}} l(x(t), u(t)) d t
$$

(as in the rest of the paper, $T_{x}$ denotes the exit-time of $x$ from $\mathbb{R}^{3} \backslash\{0\}$ ). Set

$$
f(x, u):=\left(u_{1}, u_{2}, x_{1} u_{2}-x_{2} u_{1}\right) \quad \forall(x, u) \in \mathbb{R}^{3} \times U .
$$

The following map $W_{1}$, introduced in [21], given by

$$
W_{1}(x):=\left(\sqrt{x_{1}^{2}+x_{2}^{2}}-\left|x_{3}\right|\right)^{2}+x_{3}^{2} \quad \forall x \in \mathbb{R}^{3},
$$

is proper, positive definite, locally semiconcave in $\mathbb{R}^{3} \backslash\{0\}$, and verifies

$$
\min _{u \in U}\langle p, f(x, u)\rangle=-\sqrt{V(x)} \quad \forall x \in \mathbb{R}^{3} \backslash\{0\}, \forall p \in D^{*} W_{1}(x),
$$

where

$$
V(x):=\left(\sqrt{x_{1}^{2}+x_{2}^{2}}-\left|x_{3}\right|\right)^{2}+\left(\sqrt{x_{1}^{2}+x_{2}^{2}}-2\left|x_{3}\right|\right)^{2}\left(x_{1}^{2}+x_{2}^{2}\right) \quad \forall x \in \mathbb{R}^{3} .
$$

Therefore, $W_{1}$ is a Control Lyapunov Function for the control system (5.1) and, consequently, any $W_{1}$-feedback sample and Euler stabilizes (5.1) to the origin [29]. When the Lagrangian $l$ satisfies, for some positive constant $C$,

$$
0 \leq l(x, u) \leq C \sqrt{V(x)} \quad \forall(x, u) \in\left(\mathbb{R}^{3} \backslash\{0\}\right) \times U,
$$

then $W_{1}$ is also a $p_{0}$-MRF for every $p_{0} \in(0,1 / C)$. Indeed, for all $x \in \mathbb{R}^{3} \backslash\{0\}$ and for all $p \in D^{*} W_{1}(x)$, one has

$$
\begin{gathered}
\quad=\min _{u \in U}\left\{\langle p, f(x, u)\rangle+p_{0} l(x, u)\right\} \\
\leq \min _{u \in U}\{\langle p, f(x, u)\rangle\}+p_{0} C \sqrt{V(x)}=-\left(1-p_{0} C\right) \sqrt{V(x)} .
\end{gathered}
$$

However, the Control Lyapunov Function $W_{1}$ cannot be a $p_{0}-\mathrm{MRF}$ when

$$
\lim _{x \rightarrow 0} \frac{\inf _{u \in U} l(x, u)}{\sqrt{V(x)}}=+\infty .
$$


Since $V(x)$ tends to $0^{+}$as $x \rightarrow 0$, condition (5.4) does not hold, for instance, for the minimum time problem, where $l \equiv 1$.

For any bounded Lagrangian $l$, let $M_{l}>0$ verify $l(x, u) \leq M_{l}$ for all $(x, u) \in\left(\mathbb{R}^{n} \backslash \mathbf{C}\right) \times U$. Then a discontinuous feedback that sample and Euler stabilizes (5.1) and at the meantime provides strategies for which the target is reached with regulated cost, can be obtained if we consider the following Control Lyapunov Function $W_{2}$, introduced in [27]:

$$
W_{2}(x):=\max \left\{\sqrt{x_{1}^{2}+x_{2}^{2}},\left|x_{3}\right|-\sqrt{x_{1}^{2}+x_{2}^{2}}\right\} \quad \forall x \in \mathbb{R}^{3} .
$$

The map $W_{2}$ is locally semiconcave only outside the set $S:=\left\{\left(x_{1}, x_{2}, x_{3}\right) \in\right.$ $\left.\mathbb{R}^{3} \mid x_{3}^{2}=4\left(x_{1}^{2}+x_{2}^{2}\right)\right\}$, therefore, it is not a $p_{0}$-MRF. However, $W_{2}$ matches the weaker definition of Lipschitz continuous $p_{0}-\mathrm{MRF}$ for $p_{0}<1 / M_{l}$ : it is indeed a locally Lipschitz continuous map in $\mathbb{R}^{3}$, which is positive definite and proper in $\mathbb{R}^{3} \backslash\{0\}$, and a direct computation shows that

$$
H\left(x, p_{0}, p\right) \leq \min _{u \in U}\langle f(x, u), p\rangle+p_{0} M_{l}<0 \quad \forall x \in \mathbb{R}^{3} \backslash\{0\}, \forall p \in \partial_{P} W(x)
$$

(see also [29] and [21]). Since the data $f$ and $l$ verify assumption (H1), it follows by Corollary 4.4 that (5.1)-(5.2) is sample and Euler stabilizable with $\left(\bar{l} / 2, W_{2}\right)$-regulated cost as soon as the Lagrangian $l$ is bounded.

\section{Conclusions}

In this paper we addressed sample and Euler stabilizability of nonlinear control system in an optimal control theoretic framework. We introduced the notion of sample and Euler trajectories with regulated cost, which conjugate stabilizability with an upper bound on the payoff, depending on the initial state. Under mild regularity hypotheses on the vector field $f$ and on the Lagrangian $l$ and for a closed, possibly unbounded control set, we proved that the existence of a special Control Lyapunov Function $W$, called a $p_{0}$-Minimum Restraint Function, $p_{0}-\mathrm{MRF}$, implies that all sample and Euler stabilizing trajectories have $\left(p_{0}, W\right)$-regulated costs. The proof is constructive: it is based indeed on the synthesis of appropriate feedbacks derived from $W$. As in the case of classical Control Lyapunov Functions, this construction requires that $W$ is locally semiconcave. However, by generalizing an earlier result by Rifford [28] we established that it is possible to trade regularity assumptions on $f$ and $l$ with milder regularity assumptions on $W$. In particular, we showed that if the vector field $f$ and the Lagrangian $l$ are locally Lipschitz up to the boundary of the target, then the existence of a mere locally Lipschitz $p_{0}-\mathrm{MRF} W$ provides sample and Euler stabilizability with $\left(p_{0} / 2, W\right)$-regulated cost.

The present work is part of an ongoing, wider investigation of global asymptotic controllability and stabilizability in an optimal control perspective. A slightly weaker notion of $p_{0}-\mathrm{MRF}$ - called here $p_{0}$-OMRF — was introduced in [23] and further extended in [19] to more general optimization problems, in order to yield global asymptotic controllability with regulated cost. This paper represents the stability-oriented counterpart of [23]. In a forthcoming paper we 
will address the question of stabilizability with regulated cost for possibly noncoercive optimization problems with unbounded controls. Other interesting research directions include the relation between $p_{0}$-MRFs and input-to-state stability (in the fashion of [21]) and the study of a possible inverse Lyapunov theorem for $p_{0}$-MRFs - i.e., whether the results in [30] can be extended to $p_{0}$-MRFs by showing that their existence is also a necessary condition for the global asymptotic controllability of the control system with regulated cost.

\section{Acknowledgements}

Open access funding provided by Università degli Studi di Padova within the CRUI-CARE Agreement.

Open Access. This article is licensed under a Creative Commons Attribution 4.0 International License, which permits use, sharing, adaptation, distribution and reproduction in any medium or format, as long as you give appropriate credit to the original author(s) and the source, provide a link to the Creative Commons licence, and indicate if changes were made. The images or other third party material in this article are included in the article's Creative Commons licence, unless indicated otherwise in a credit line to the material. If material is not included in the article's Creative Commons licence and your intended use is not permitted by statutory regulation or exceeds the permitted use, you will need to obtain permission directly from the copyright holder. To view a copy of this licence, visit http://creativecommons. org/licenses/by/4.0/.

Publisher's Note Springer Nature remains neutral with regard to jurisdictional claims in published maps and institutional affiliations.

\section{Appendix A.}

\section{A.1. Proof of Theorem 4.3}

Let $W: \overline{\mathbb{R}^{n} \backslash \mathbf{C}} \rightarrow[0,+\infty)$ be a locally Lipschitz continuous map, positive definite and proper on $\mathbb{R}^{n} \backslash \mathbf{C}$, and verifying the decrease condition (4.3), namely, such that

$$
H\left(x, p_{0}, \partial_{P} W(x)\right) \leq-\tilde{\gamma}\left(W_{1}(x)\right) \quad \forall x \in \mathbb{R}^{n} \backslash \mathbf{C}
$$

for some strictly increasing, continuous map $\tilde{\gamma}:(0,+\infty) \rightarrow(0,+\infty)$ and some continuous function $W_{1}: \overline{\mathbb{R}^{n} \backslash \mathbf{C}} \rightarrow[0,+\infty), W_{1}$ positive definite and proper on $\mathbb{R}^{n} \backslash$ C. Our goal is to show that there exists a $\frac{p_{0}}{2}-\mathrm{MRF} \bar{W}$, such that $\bar{W} \leq W$.

The proof is a careful adaptation of the arguments in [28, sect. 5]. For this reason we explicitly prove just the steps involving the decrease condition, where some changes are needed because of the presence of the Lagrangian $l$.

Preliminarily, let us recall the notion of inf-convolution for a locally Lipschitz continuous nonnegative map $g: \mathbb{R}^{N} \rightarrow \mathbb{R}$ and collect some useful properties (see e.g. [9, Theorem 3.5.3, Lemma 3.5.7], [12, Section 1.5, Thm. 5.1], [5, Section II.4, Lemmas 4.11, 4.12]). 
Lemma A.1. For any $\alpha>0$, define

$$
g_{\alpha}(x):=\inf _{y \in \mathbb{R}^{N}}\left\{g(y)+\alpha|y-x|^{2}\right\} \quad \forall x \in \mathbb{R}^{N} .
$$

Then $g_{\alpha}$ is locally semiconcave in $\mathbb{R}^{N}$ and

(i) for all $x \in \mathbb{R}^{N}$, there exists $\bar{y} \in \mathbb{R}^{N}$ such that $g_{\alpha}(x)=g(\bar{y})+\alpha|\bar{y}-x|^{2}$ (the above infimum is actually a minimum);

(ii) for all $x \in \mathbb{R}^{N}, 0 \leq g_{\alpha}(x) \leq g(x)$, moreover $g_{\alpha} \nearrow g$ locally uniformly as $\alpha \rightarrow 0$;

(iii) for all $x \in \mathbb{R}^{N}$ such that $\partial_{P} g_{\alpha}(x)$ is nonempty, $\bar{y}$ is unique and the proximal subgradient $\partial_{P} g_{\alpha}(x)$ is equal to the singleton $\{2 \alpha(x-\bar{y})\}$, moreover, $2 \alpha(x-\bar{y}) \in \partial_{P} g(\bar{y})$;

(iv) if $\Psi: \mathbb{R} \rightarrow \mathbb{R}$ is an increasing, locally semiconcave function, then $\Psi \circ g_{\alpha}$ is locally semiconcave;

(v) if $g, h: \mathbb{R}^{N} \rightarrow \mathbb{R}$ are semiconcave on $\Omega \subset \mathbb{R}^{N}$, then the function $\min \{g, h\}$ is semiconcave on $\Omega$.

STEP 1. As it is not restrictive in view of Proposition 4.2, let us assume that $W_{1} \equiv \mathbf{d}$. We extend $\tilde{\gamma}$ continuously to $\mathbb{R}$, by setting $\tilde{\gamma}(t)=\tilde{\gamma}(0):=$ $\lim _{s \rightarrow 0^{+}} \tilde{\gamma}(s)$ for every $t<0$. Without loss of generality, we can suppose $\tilde{\gamma}$ 1-Lipschitz continuous in $\mathbb{R}$. Otherwise, we can replace $\tilde{\gamma}$ in the decrease condition (A.1) with $\bar{\gamma}(t):=\inf _{s \in \mathbb{R}}\{\tilde{\gamma}(s)+|t-s|\}$ for every $t \in \mathbb{R}$. Indeed, it is not difficult to see that $\bar{\gamma} \leq \tilde{\gamma}$, and $\bar{\gamma}$ is strictly increasing and 1-Lipschitz continuous. Therefore, the map

$$
\mathcal{W}:=\bar{\gamma} \circ W_{1}=\bar{\gamma} \circ \mathbf{d}
$$

is 1-Lipschitz continuous and positive definite on $\mathbb{R}^{n} \backslash \mathbf{C}$. As a consequence of these results, (A.1) implies that $W$ verifies

$$
H\left(x, p_{0}, \partial_{P} W(x)\right) \leq-\mathcal{W}(x) \quad \forall x \in \mathbb{R}^{n} \backslash \mathbf{C} .
$$

STEP 2. For any integer $n \geq 1$, let us set

$$
\begin{aligned}
M_{n} & :=\max \left\{W(x): x \in B_{1}\left(W^{-1}([0,11 n])\right)\right\}, \\
m_{n} & :=\min \left\{\mathcal{W}(x): x \in W^{-1}\left(\left[\frac{1}{2 n}, 11 n\right]\right)\right\} .
\end{aligned}
$$

By the Lipschitz properties of $f, l$ and $W$, let us denote $L_{f}^{n}, L_{l}^{n}, L_{W}^{n} \geq 1$ the Lipschitz constants of $f(\cdot, u), l(\cdot, u)$ and $W$, respectively, on the sublevel set $W^{-1}\left(\left[0, M_{n}\right]\right)$. Finally, let us set

$$
\alpha_{n}:=\max \left\{8 n\left(L_{W}^{n}\right)^{2}+1, \frac{2 L_{W}^{n}\left(1+L_{W}^{n} L_{f}^{n}+p_{0} L_{l}^{n}\right)}{m_{n}}+1,11 n\right\} .
$$

Let us extend $W$ to $\mathbb{R}^{n}$ by setting $W(x)=0$ for all $x$ in the interior of $\mathbf{C}$. For every $\alpha_{n}$, we define by inf-convolution the locally semiconcave function $W_{\alpha_{n}}: \mathbb{R}^{n} \rightarrow[0,+\infty)$ as follows:

$$
W_{\alpha_{n}}(x):=\inf _{y \in \mathbb{R}^{n}}\left\{W(y)+\alpha_{n}|y-x|^{2}\right\} \quad \forall x \in \mathbb{R}^{n} .
$$


Lemma A.2. ( $\left[28\right.$, Lemma 5.5]) Let $z \in W^{-1}\left(\left[0, M_{n}\right]\right)$. If the infimum in the definition of $W_{\alpha_{n}}(z)$ is attained at $\bar{y}$, then one has that $\bar{y} \in W^{-1}\left(\left[0, M_{n}\right]\right)$ and $|\bar{y}-z| \leq \min \left\{\frac{1}{8 n L_{W}^{n}}, \frac{m_{n}}{2\left(1+L_{W}^{n} L_{f}^{n}+p_{0} L_{l}^{n}\right)}\right\} ;$ moreover

$$
W(z)-\frac{1}{8 n} \leq W_{\alpha_{n}}(z) \leq W(z) .
$$

Lemma A.3. Let $z \in W^{-1}\left(\left[\frac{1}{2 n}, 11 n\right]\right)$ and $p \in \partial_{P} W_{\alpha_{n}}(z)$. Then

$$
H\left(z, p_{0}, p\right) \leq-\frac{\mathcal{W}(z)}{2}
$$

Proof. Arguing similarly to the proof of [28, Lemma 5.6], by Lemmas A.2 and A.1, the infimum in the definition of $W_{\alpha_{n}}(z)$ is attained at a point $\bar{y} \in$ $W^{-1}([0,11 n])$, verifying $|\bar{y}-z| \leq \frac{m_{n}}{2\left(1+L_{W}^{n} L_{f}^{n}+p_{0} L_{l}^{n}\right)}$ and such that $p \in \partial_{P} W(\bar{y})$. Therefore, by the Lipschitz properties of $f, l, W$ and the 1-Lipschitz continuity of $\mathcal{W}$ established in Step 1, we get

$$
\begin{aligned}
H\left(z, p_{0}, p\right)= & \inf _{u \in U}\left\{\langle p, f(z, u)\rangle+p_{0} l(z, u)\right\} \leq \inf _{u \in U}\left\{\langle p, f(\bar{y}, u)\rangle+p_{0} l(\bar{y}, u)\right\} \\
& +\sup _{u \in U}\left(|p||f(z, u)-f(\bar{y}, u)|+p_{0}|l(z, u)-l(\bar{y}, u)|\right) \\
\leq & -\mathcal{W}(\bar{y})+L_{W}^{n} L_{f}^{n}|z-\bar{y}|+p_{0} L_{l}^{n}|z-\bar{y}| \quad(\operatorname{using}(\mathrm{A} .2)) \\
\leq & -\mathcal{W}(z)+\left(1+L_{W}^{n} L_{f}^{n}+p_{0} L_{l}^{n}\right)|z-\bar{y}| \\
\leq & -\mathcal{W}(z)+\frac{m_{n}}{2} \leq-\frac{\mathcal{W}(z)}{2} .
\end{aligned}
$$

STEP 3. Starting from $\left(W_{\alpha_{n}}\right)_{n \geq 1}$, let us construct a locally semiconcave $\frac{p_{0}}{2}-\mathrm{MRF}$.

Lemma A.4. ( [28, Lemma 5.7]) For each $n \geq 1$, there exists an increasing, $C^{\infty}$, increasing map $\left.\Psi_{n}:[0,+\infty) \rightarrow: 0,+\infty\right)$ verifying the following properties.

(i) $\Psi_{n}(t)=t+\frac{1}{8 n}$ for any $t \in\left[0, \frac{1}{2 n}\right]$,

(ii) $\Psi_{n}(t)=t$ for any $t \in\left[\frac{1}{n}-\frac{1}{8 n}, 10 n\right]$,

(iii) $\Psi_{n}(t) \geq 11 n+\max \left\{W(x): W_{\alpha_{n}}(x) \leq t\right\}$ for any $t \in\left[11 n-\frac{1}{8 n},+\infty\right)$,

(iv) $\dot{\Psi}_{n}(t) \geq \frac{1}{2}$ for any $t \geq 0$.

The function $\bar{W}_{n}:=\Psi_{n} \circ W_{\alpha_{n}}$ is locally semiconcave on $\mathbb{R}^{n}$ by Lemma A.1, (iv). The required locally semiconcave $\frac{p_{0}}{2}-\mathrm{MRF} \bar{W}$, is given by

$$
\bar{W}(x):=\min _{n \geq 1} \bar{W}_{n}(x) \quad \forall x \in \mathbb{R}^{n} \backslash \mathbf{C} .
$$

Precisely, one has what follows.

Lemma A.5. For all integer $n \geq 1$ and for all $z \in W^{-1}\left(\left[\frac{1}{n}, 10 n\right]\right)$, one has $\bar{W}(z)=\min _{1 \leq k \leq n} \bar{W}_{k}(z)$. Furthermore, if $p \in \partial_{P} \bar{W}(z)$, then

$$
H\left(z, \frac{p_{0}}{2}, p\right) \leq-\frac{\mathcal{W}(z)}{4} .
$$


Proof. For all $z \in W^{-1}\left(\left[\frac{1}{n}, 10 n\right]\right)$, the proof of the following facts:

(i) $\bar{W}(z)=\bar{W}_{n_{0}}(z):=\min _{1 \leq k \leq n} \bar{W}_{k}(z)$ and

$$
\left.p \in \partial_{P} \bar{W}(z) \Longrightarrow p \in \partial_{P} \bar{W}_{n_{0}}(z)=\Psi_{n_{0}}^{\prime}\left(W_{\alpha_{n_{0}}}(z)\right) \partial_{P} W_{\alpha_{n_{0}}}(z)\right) ;
$$

(ii) $W(z) \leq 11 n_{0}$,

can be derived easily by [28, Lemma 5.8], hence we omit it. If $W(z)<\frac{1}{2 n_{0}}$, then

$$
W_{n_{0}}(z) \leq W(z)<\frac{1}{2 n_{0}} \Longrightarrow \bar{W}_{n_{0}}(z)=W_{\alpha_{n_{0}}}(z)+\frac{1}{8 n_{0}} \geq W(z) .
$$

Since $\bar{W}_{n}(z)=W_{\alpha_{n}}(z) \leq W(z)$, this yields that the minimum is also reached for $n$. Thus Lemma A.4 (i) and Lemma A.3 imply the decrease condition (A.6) for any $p \in \partial_{P} \bar{W}(z)$. It remains to show that (A.6) holds for any $p \in \partial_{P} \bar{W}(z)$ also when $z \in W^{-1}\left(\left[\frac{1}{2 n_{0}}, 11 n_{0}\right]\right)$. By Lemma A.3,

$$
H\left(z, p_{0}, p\right)=\inf _{u \in U}\left\{\langle p, f(z, u)\rangle+p_{0} l(z, u)\right\} \leq-\frac{\mathcal{W}(z)}{2} \quad \forall p \in \partial_{P} W_{\alpha_{n_{0}}}(z)
$$

and, as a consequence (since $p_{0} \geq 0$ and $l \geq 0$ ),

$$
\inf _{u \in U}\{\langle p, f(z, u)\rangle\}<0 \quad \forall p \in \partial_{P} W_{\alpha_{n_{0}}}(z) .
$$

Thus by Lemma A.4 (iv) and (A.7), for any $p \in \partial_{P} \bar{W}(z)$ there is some $p_{n_{0}} \in$ $\partial_{P} W_{\alpha_{n_{0}}}(z)$ such that $p=\Psi_{n_{0}}^{\prime}\left(W_{\alpha_{n_{0}}}(z)\right) p_{n_{0}}$ and

$$
\begin{aligned}
H\left(z, \frac{p_{0}}{2}, p\right) & =\inf _{u \in U}\left\{\langle p, f(z, u)\rangle+\frac{p_{0}}{2} l(z, u)\right\} \\
& =\inf _{u \in U}\left\{\Psi_{n_{0}}^{\prime}\left(W_{\alpha_{n_{0}}}(z)\right)\left\langle p_{n_{0}}, f(z, u)\right\rangle+\frac{p_{0}}{2} l(z, u)\right\} \\
& \leq \frac{1}{2} H\left(z, p_{0}, p\right) \leq-\frac{\mathcal{W}(z)}{4} .
\end{aligned}
$$

This last lemma shows that the minimum in the definition of $\bar{W}(x)$ is always attained for $x \in \mathbb{R}^{n} \backslash \mathbf{C}$. Therefore, the function $\bar{W}$ is locally semiconcave outside the target (by Lemma A.1, (v)). On the other hand, $\bar{W}$ is continuous on $\overline{\mathbb{R}^{n} \backslash \mathbf{C}}$ because $0 \leq \bar{W} \leq W$ and satisfies the decrease condition by (A.6), where, by Step $1, \frac{\mathcal{W}}{4}$ coincides with the composition of the positive, Lipschitz continuous, and strictly increasing function $\frac{\bar{\gamma}}{4}$ with the distance d. Consequently, by Proposition 4.1 we can conclude that $\bar{W}$ provides a $\frac{p_{0}}{2}$-MRF, which proves Theorem 4.3.

\section{A.2. Proof of Theorem 4.9}

Let us sketch how to adapt the arguments of the proof of Theorem 4.3 to the case of a Lipschitz continuous $p_{0}$-OMRF, for which only the existence of a local rate function $\gamma_{\sigma}$ is ensured.

Let $W: \overline{R^{n} \backslash \mathbf{C}} \rightarrow[0,+\infty)$ be a locally Lipschitz continuous map, positive definite and proper on $\mathbb{R}^{n} \backslash \mathbf{C}$ and verifying

$$
H\left(x, p_{0}, \partial_{L} W(x)\right)<0 \quad \forall x \in \mathbb{R}^{n} \backslash \mathbf{C} .
$$

Our aim is to prove the existence of a $\frac{p_{0}}{2}$-OMRF $\bar{W} \leq W$. 
SteP 1. By the Lipschitz continuity of $W$, the set-valued map $x \rightsquigarrow$ $\partial_{L} W(x)$ has closed graph with compact values, so that it is upper semicontinuous (see [35, Props. 4.3.3, 4.3.5] and [2, Thm.1 and Cor. 1, pg. 41]). At this point, one can derive that for any $\sigma>0$ there exists a positive, continuous, strictly increasing map $\gamma_{\sigma}$ defined in $(0, \sigma]$, such that $W$ verifies

$$
H\left(x, p_{0}, D_{L} W(x)\right)<-\gamma_{\sigma}(W(x)) \quad \forall x \in W^{-1}((0, \sigma]),
$$

arguing exactly as in [23, Prop. 3.1]. If $\gamma_{\sigma}$ denotes an arbitrary continuous and strictly increasing extension of $\gamma_{\sigma}$ to $(0,+\infty)$, by Proposition 4.2 it follows that for any continuous function $W_{1}: \overline{\mathbb{R}^{n} \backslash \mathbf{C}} \rightarrow[0,+\infty)$, positive definite and proper on $\mathbb{R}^{n} \backslash \mathbf{C}$, there exists a continuous, strictly increasing function $\tilde{\gamma}_{\sigma}:(0,+\infty) \rightarrow(0,+\infty)$ such that $W$ also verifies

$$
H\left(x, p_{0}, \partial_{L} W(x)\right) \leq-\tilde{\gamma}_{\sigma}\left(W_{1}(x)\right) \quad \forall x \in W^{-1}((0, \sigma])
$$

(and vice-versa, if $W, W_{1}$ satisfy (A.9), then (A.8) holds true for some $\gamma_{\sigma}$ as above). Let us choose $W_{1}=\mathbf{d}$. Similarly to Step 1 of the proof of Theorem 4.3, we can suppose without loss of generality that $\tilde{\gamma}_{\sigma}$ is 1-Lipschitz continuous and consider the map $\mathcal{W}_{\sigma}:=\tilde{\gamma}_{\sigma} \circ \mathbf{d}$, which is 1-Lipschitz continuous and positive definite in $\mathbb{R}^{n} \backslash \mathbf{C}$. Therefore, recalling that $\partial_{P} W(x) \subseteq \partial_{L} W(x)$ for every $x$, $W$ verifies

$$
H\left(x, p_{0}, \partial_{P} W(x)\right) \leq-\mathcal{W}_{\sigma}(x) \quad \forall x \in W^{-1}((0, \sigma]) .
$$

STEP 2. For any integer $n \geq 1$, let us set $\sigma_{n}:=11 n$. Hence $W$ verifies

$$
H\left(x, p_{0}, \partial_{P} W(x)\right) \leq-\mathcal{W}_{\sigma_{n}}(x) \quad \forall x \in W^{-1}\left(\left(0, \sigma_{n}\right]\right),
$$

where it is easy to see that $\left(\mathcal{W}_{\sigma_{n}}\right)_{n}$ is a decreasing sequence. From now on, the proof proceeds similarly to "Appendix A.1", with the crucial differences that the decrease rate $\mathcal{W}_{\sigma_{n}}$ in (A.11) depends on $\sigma_{n}$ and that the condition (A.11) is satisfied only in $W^{-1}\left(\left(0, \sigma_{n}\right]\right)$. In particular, these facts imply that, for any $n \geq 1$, the inf-convolution $W_{\alpha_{n}}$ of $W$ depends on $\mathcal{W}_{\sigma_{n}}$, since $\alpha_{n}$ is given by

$$
\alpha_{n}:=\max \left\{8 n\left(L_{W}^{n}\right)^{2}+1, \frac{2 L_{W}^{n}\left(1+L_{W}^{n} L_{f}^{n}+p_{0} L_{l}^{n}\right)}{m_{n}}+1,11 n\right\},
$$

where all the constants are the same as in the proof of Theorem 4.3 and, in particular,

$$
m_{n}=\min \left\{\mathcal{W}_{\sigma_{n}}(x): \quad x \in W^{-1}\left(\left[\frac{1}{2 n}, 11 n\right]\right)\right\} .
$$

Lemmas A.2, A.4, dealing with the properties of the approximations of $W$, hold unchanged, while Lemmas A.3, A.5 are now replaced by the following results.

Lemma A.6. Let $z \in W^{-1}\left(\left[\frac{1}{2 n}, \sigma_{n}\right]\right)$ and $p \in \partial_{P} W_{\alpha_{n}}(z)$. Then

$$
H\left(z, p_{0}, p\right) \leq-\frac{\mathcal{W}_{\sigma_{n}}(z)}{2} \text {. }
$$


Proof. The only delicate point in order to adapt the proof of Lemma A.3 to the present setting, is that, given $z \in W^{-1}\left(\left(0, \sigma_{n}\right]\right)$, one has to apply the decrease condition in (A.11) not at $z$, but at the point $\bar{y}$ where the minimum in definition (A.4) of $W_{\alpha_{n}}(x)$ is obtained. This can be done since $\bar{y}$ belongs to the sublevel set $W^{-1}\left(\left(0, \sigma_{n}\right]\right)$ too; indeed,

$$
W(\bar{y})=W_{\alpha_{n}}(z)-\alpha_{n}|\bar{y}-z|^{2} \leq W_{\alpha_{n}}(z) \leq W(z) \leq \sigma_{n} .
$$

Lemma A.7. For all integer $n \geq 1$ and for all $z \in W^{-1}\left(\left[\frac{1}{n}, \sigma_{n}-n\right]\right)$, one has $\bar{W}(z)=\bar{W}_{n_{0}}(z)$ for some $n_{0} \in\{1, \ldots, n\}$. Furthermore, if $p \in \partial_{P} \bar{W}(z)$, then

$$
H\left(z, \frac{p_{0}}{2}, p\right) \leq-\frac{\mathcal{W}_{\sigma_{n}}(z)}{4} .
$$

Proof. Going through the proof of Lemma A.5, the crucial remark is that, whenever the minimum

$$
\bar{W}(z)=\bar{W}_{n_{0}}(z):=\min _{1 \leq k \leq n} \bar{W}_{n}(z)
$$

is obtained for some $n_{0}<n$, then $W(z) \leq \sigma_{n_{0}}$. The last inequality implies that, when $W(z) \geq \frac{1}{2 n_{0}}$, the point $z$ belongs to the strip $W^{-1}\left(\left[\frac{1}{2 n_{0}}, \sigma_{n_{0}}\right]\right)$. Therefore, arguing as in the proof of Lemma A.5, one can apply Lemma A.6 to derive that

$$
H\left(z, \frac{p_{0}}{2}, p\right) \leq-\frac{\mathcal{W}_{\sigma_{n_{0}}}(z)}{4} .
$$

Recalling that the sequence $\left(\mathcal{W}_{\sigma_{n}}\right)$ is decreasing, this yields the decrease condition (A.13). The proof in the case $W(z)<\frac{1}{2 n_{0}}$, where one can assume $n_{0}=n$, can be obtained again by Lemma A.6 and the arguments of the proof of Lemma A.5.

The decrease condition (4.4) follows now by the arbitrariness of $n$ and, consequently, we have that $\bar{W}$ is a $p_{0} / 2-\mathrm{OMRF}$.

\section{References}

[1] Artstein, Z.: Stabilization with relaxed controls. Nonlinear Anal. 7(11), 1163$1173(1983)$

[2] Aubin, J.P., Cellina, A.: Differential Inclusions. Set-Valued Maps and Viability Theory, Grundlehren der Mathematischen Wissenschaften [Fundamental Principles of Mathematical Sciences], 264. Springer, Berlin (1984)

[3] Bacciotti, A., Rosier, L.: Lyapunov Functions and Stability in Control Theory. Lecture Notes in Control and Information Sciences, vol. 267. Springer, London (2001)

[4] Bao, X., Lin, Z.: On $L^{p}$ input to state stabilizability of affine in control, nonlinear systems subject to actuator saturation. J. Franklin Inst. 337, 691-712 (2000) 
[5] Bardi, M., Dolcetta, I.: Capuzzo: Optimal Control and Viscosity Solutions of Hamilton-Jacobi-Bellman Equations. Birkhäuser, Boston (1997)

[6] Bressan, A., Rampazzo, F.: Moving constraints as stabilizing controls in classical mechanics. Archive Ration. Mech. Anal. 196(1), 97-141 (2010)

[7] Brockett, R.W.: Asymptotic stability and feedback stabilization. Differ. Geomet. Control Theory 27(1), 181-191 (1983)

[8] Camilli, F., Siconolfi, A.: Maximal subsolution for a class of degenerate Hamilton-Jacobi problems. Indiana Univ. Math. J. 48, 1111-1131 (1999)

[9] Cannarsa, P., Sinestrari, C.: Semiconcave Functions, Hamilton-Jacobi Equations, and Optimal Control, Progress in Nonlinear Differential Equations and Their Applications, 58. Birkhäuser, Boston (2004)

[10] Chitour, Y.: On the $L^{p}$ stabilization of the double integrator subject to input saturation. ESAIM Control Optim. Calc. Var. 6, 291-331 (2001)

[11] Chitour, Y., Liu, W., Sontag, E.: On finite-gain stabilizability of linear systems subject to input saturation. SIAM J. Control Optim. 34, 1190-1219 (1996)

[12] Clarke, F.H., Ledyaev, YuS, Stern, R.J., Wolenski, P.R.: Nonsmooth Analysis and Control Theory, Graduate Texts in Mathematics, 178. Springer, New York (1998)

[13] Clarke, F., Ledyaev, Y., Sontag, E., Subbotin, A.: Asymptotic controllability implies feedback stabilization. IEEE Trans. Automat. Control 42(10), 1394-1407 (1997)

[14] Clarke, F., Ledyaev, Y., Rifford, L., Stern, R.J.: Feedback stabilization and Lyapunov functions. SIAM J. Control Optim. 39, 25-48 (2000)

[15] Coron, J.-M., Praly, L., Teel, A.: Feedback Stabilization of Nonlinear Systems: Sufficient Conditions and Lyapunov and Input-Output Techniques. Trends in control, pp. 293-348. Springer, London (1995)

[16] Ishii, H., Ramaswamy, M.: Uniqueness results for a class of Hamilton-Jacobi equations with singular coefficients. Commun. Partial Differ. Equ. 20, 2187-2213 (1995)

[17] Krasovskii, N.N., Subbotin, A.I.: Game-Theoretical Control Problems. Springer, New York (1988)

[18] Lai, A. C., Motta, M.: Stabilizability in impulsive optimization problems, IFACPapersOnLine, 52(16), Proceedings of the 11th IFAC Symposium on Nonlinear Control Systems, NOLCOS, Vienna, pp. 352-357 (2019)

[19] Lai, A.C., Motta, M., Rampazzo, F.: Minimum restraint functions for unbounded dynamics: general and control-polynomial systems. Pure Appl. Funct. Anal. 1(4), 583-612 (2016)

[20] Malisoff, M.: Bounded-from-below solutions of the Hamilton-Jacobi equation for optimal control problems with exit times: vanishing Lagrangians, eikonal 
equations, and shape-from-shading. NoDEA Nonlinear Differ. Equ. Appl. 11, 95-122 (2004)

[21] Malisoff, M., Rifford, L., Sontag, E.: Global asymptotic controllability implies input-to-state stabilization. SIAM J. Control Optim. 42(6), 2221-2238 (1997)

[22] Motta, M.: Viscosity solutions of HJB equations with unbounded data and characteristic points. Appl. Math. Optim. 49(1), 1-26 (2004)

[23] Motta, M., Rampazzo, F.: Asymptotic controllability and optimal control. J. Differ. Equ. 254(7), 2744-2763 (2013)

[24] Motta, M., Sartori, C.: On asymptotic exit-time control problems lacking coercivity. ESAIM Control Optim. Calc. Var. 20(4), 957-982 (2014)

[25] Motta, M., Sartori, C.: The value function of an asymptotic exit-time optimal control problem. NoDEA Nonlinear Differ. Equ. Appl. 22(1), 21-44 (2015)

[26] Motta, M., Sartori, C.: Asymptotic problems in optimal control with a vanishing Lagrangian and unbounded data. Discrete Contin. Dyn. Syst. 35(9), 4527-4552 (2015)

[27] Rifford, L.: Problèmes de Stabilisation en Théorie du Controle. Université Claude Bernard Lyon I, Lyon (2000). Ph.D. thesis

[28] Rifford, L.: Existence of Lipschitz and semiconcave control-Lyapunov functions and stabilizing feedbacks. SIAM J. Control Optim. 39(4), 1043-1064 (2000)

[29] Rifford, L.: Semiconcave control-Lyapunov functions and stabilizing feedbacks. SIAM J. Control Optim. 41(3), 659-681 (2002)

[30] Sontag, E.D.: A Lyapunov-like characterization of asymptotic controllability. SIAM J. Control Optim. 21(3), 462-471 (1983)

[31] Sontag, E., Sussmann, H.J.: Remarks on continuous feedback. In: 1980 19th IEEE Conference on Decision and Control including the Symposium on Adaptive Processes. IEEE (1980)

[32] Sontag, E., Sussmann, H.J.: Nonsmooth control-Lyapunov functions. In: Proceedings of 1995 34th IEEE Conference on Decision and Control. Vol. 3. IEEE (1995)

[33] Soravia, P.: Optimality principles and representation formulas for viscosity solutions of Hamilton-Jacobi equations I: equations of unbounded and degenerate control problems without uniqueness. Adv. Differ. Equ. 275-296 (1999)

[34] Sussmann, H., Sontag, E.D., Yang, Y.: A general result on the stabilization of linear systems using bounded controls. IEEE Trans. Automat. Control 39, 2411-2425 (1994)

[35] Vinter, R.B.: Optimal Control. Birkhäuser, Boston (2000) 
Anna Chiara Lai

Dipartimento di Scienze di Base e Applicate per l'Ingegneria

Sapienza Università di Roma

Via Scarpa 16

00181 Rome

Italy

e-mail: anna.lai@sbai.uniroma1.it

Monica Motta

Dipartimento di Matematica

Università di Padova

Via Trieste, 63

35121 Padua

Italy

e-mail: motta@math.unipd.it

Received: 26 December 2018.

Accepted: 4 July 2020. 